\title{
Analysis of Non-Stationary 3D Air-to-Air Channels Using the Theory of Algebraic Curves
}

\author{
Michael Walter, Senior Member, IEEE, Dmitriy Shutin, Senior Member, IEEE, David W. Matolak, Senior \\ Member, IEEE, Nicolas Schneckenburger, Member, IEEE, Thomas Wiedemann, Armin Dammann, Member, IEEE
}

\begin{abstract}
Non-stationary channel models play a crucial role in today's communication systems. Mobile-to-mobile channels are known to exhibit non-stationary behavior caused by the movement of transmitter and receiver. Non-stationarity can be addressed by introducing time-variant stochastic functions such as the time-variant instantaneous Doppler probability density function or time-variant instantaneous characteristic function. An algebraic analysis of time-variant Doppler probability density function (pdf) in a classical Cartesian coordinate system is only numerically tractable due to trigonometric functions in the resulting expressions. In contrast, it has been shown that using prolate spheroidal coordinates for $2 \mathrm{D}$ vehicle-to-vehicle channels the algebraic analysis becomes analytically tractable. In this paper, the analysis is extended to aircraft-to-aircraft channels. It is shown that the time-variant Doppler pdf can be represented without trigonometric functions. The description of the Doppler frequency in prolate spheroidal coordinates allows to describe it as an algebraic curve. This permits the use of algebraic methods to analyze the Doppler frequency and derive the boundaries of the resulting Doppler pdf. Using the developed tools, we investigated exemplary aircraft-to-aircraft scenarios. However, the methodology can be extended to any aircraft-toaircraft configuration.
\end{abstract}

Index Terms-Characteristic function, Doppler pdf, mobileto-mobile channel, geometry-based stochastic channel modeling, prolate spheroidal coordinates.

\section{INTRODUCTION}

D EVICE-TO-DEVICE communication or mobile-tomobile (M2M) communication will be one of the key technologies for $5 \mathrm{G}$ communication networks [1]. An important class of M2M communication is air-to-air (A2A) communication, which is needed for both manned [2] and unmanned aerial vehicles, see [3]. Thus, a fundamental understanding of the propagation environment is crucial in order to optimally adapt new communication systems to the channel conditions. The A2A channel, however, is more complicated than the fixed-to-mobile channel of previous communication generations, e.g., 2G-4G cellular, since both transmitter (TX) and receiver (RX) are moving.

In the early days of channel modeling, mainly stochastic models were used to characterize the propagation channel [4]. Those stochastic models are based on the wide-sense stationary uncorrelated scattering (WSSUS) assumption that

M. Walter, D. Shutin, N. Schneckenburger, Thomas Wiedemann, and Armin Dammann are with the German Aerospace Center (DLR), Institute of Communications and Navigation, 82234 Wessling, Germany (email: \{m.walter, dmitriy.shutin, nicolas.schneckenburger, thomas.wiedemann, armin.dammann $\} @$ dlr.de), D. W. Matolak is with the College of Engineering and Computing, University of South Carolina, Columbia, SC 29208, USA (e-mail: matolak@cec.sc.edu). was introduced in the seminal paper of Bello [5]. This assumption is fulfilled for narrowband fixed-to-mobile channels for some limited spatial extent or temporal duration. For wideband channels the wide-sense stationary (WSS) approximation can pertain to individual multipath components as well as to the entire channel impulse response, still of course for some limited spatial extent. For azimuthally uniformly distributed scatterers, Clarke derived in [4] the so called Jakes Doppler power spectrum [6], whose inverse Fourier transform - the autocorrelation function of the channel's narrowband amplitude is a Bessel function. This description has been used for a long time for the case of a fixed base station and mobile receiver. A WSSUS model for the M2M channel was introduced in [7]. Its extensions were derived in [8]-[11] for various 2D and 3D scenarios as well as for multiple input multiple output (MIMO) scenarios in [12]-[16]. For the aeronautical channel the authors in [17] use the WSŞUS assumption when proposing aeronautical Doppler spectra. According to Bello [18], the so-called surface scatter channel for air-tosatellite communications possesses three main components: the line-of-sight (LOS) component, the specular reflection (SR) component from the earth surface, and diffuse scattering components. The Bello model applies to narrowband channels, so all diffuse components are essentially combined with the others.

For mobile-to-mobile channels, however, the WSSUS assumption is mostly violated due to the simultaneous movement of transmitter and receiver. This leads to a higher rate of time variation, which can cause non-stationarity, or a shorter stationarity time/distance. This has been observed for vehicleto-vehicle (V2V) channels according to [19] and [20]. But this observation can be extended to other M2M channel scenarios.

To address non-stationarity Matz extended Bello's model in [21] to non-WSSUS scenarios. The resulting model is represented by four-dimensional channel functions, which are unfortunately difficult to handle in practice. Following [22], who suggested to model the Doppler spectrum as timevariant function, we proposed in [23] a simpler solution by considering time-variant delay Doppler pdfs for V2V channels. Our resulting model preserves the uncorrelated scattering (US) assumption only. In [24] the proposed model was extended to A2A scenarios. Our theoretical models were verified using measurement data for both $\mathrm{V} 2 \mathrm{~V}$ in [25], [26] and $\mathrm{A} 2 \mathrm{~A}$ in [27] and [28]. Similar modeling was applied in [29]-[31] for air-to-ground (A2G) channels.

The modeling approach in the cited works on time-variant, delay-dependent Doppler pdfs was done in Cartesian coor- 
dinates. Due to the presence of mobile TX and RX the corresponding geometrical description of the channel implies a two-center problem. Therefore, a fixed delay naturally defines an ellipse for a 2D scenario and an ellipsoid for a 3D scenario, since this particular geometric shape defines the locations, where the delay between TX and RX via a scatter is constant. In the original model we inserted a parametrized ellipse into the Cartesian coordinates to obtain the results. Although tractable, the resulting models were nonetheless difficult to analyze analytically.

It is know that two-center problems are conveniently represented in prolate spheroidal coordinates. In our case the ellipse of a constant delay would thus define a single coordinate in the prolate spheroidal coordinate system (PSCS). Therefore, it was advantageous to transform the time-variant, delay-dependent Doppler pdf description into prolate spheroidal coordinates. This was done in [32] for $\mathrm{V} 2 \mathrm{~V}$ channels. We observed that the resulting models have a much simpler description of the delay-dependent Doppler frequency. This allowed us to obtain closed-form Doppler pdfs and their characteristic functions for line-of-sight and its vicinity, as well as for infinite delays in general V2V scenarios in [33]. The generalization of the results for arbitrary delays required the application of algebraic curve analysis due the ambiguities of the Doppler frequency. This only becomes apparent through the use of a PSCS and allows for an algebraic analysis of Doppler frequencies. For V2V channels this investigation was reported in [34]. The extension of these results for A2A channels is, however, still missing.

In this paper, we study the model of time-variant, delaydependent Doppler pdfs in the PSCS. Specifically, we derive the 3D time-variant, delay-dependent Doppler pdf and its Fourier dual - the characteristic function - for single-bounce scattering based on the 2D V2V model presented in [33]. We explicitly derive analytical expressions of delay-dependent characteristic functions and Doppler pdfs for the LOS component, the SR component, and the diffuse scattering components for arbitrary velocity vectors of the aircraft. Moreover, the frequencies that determine the support of the Doppler pdf, which we term the limiting frequencies, are derived and analyzed using the theory of algebraic curves. The limiting frequencies can be calculated by a sixth order polynomial for arbitrary delays for any velocity configuration. We discovered that in addition to extreme points that describe the support of the Doppler pdf, the algebraic curve analysis reveals the presence of singular points. Under specific conditions these singular points can become extreme points and have to be taken into account for calculating the limiting frequencies of the Doppler pdf.

The remainder of the paper is structured as follows. In Section II, we introduce the prolate spheroidal coordinate system and derive the Doppler pdf and characteristic function in prolate spheroidal coordinates. An algebraic analysis of the Doppler frequency leads to closed-form expressions of Doppler pdf, characteristic function, and the limiting frequencies of the Doppler pdf in Section III. The results for typical A2A scenarios are shown in Section IV and the paper is concluded with Section V.

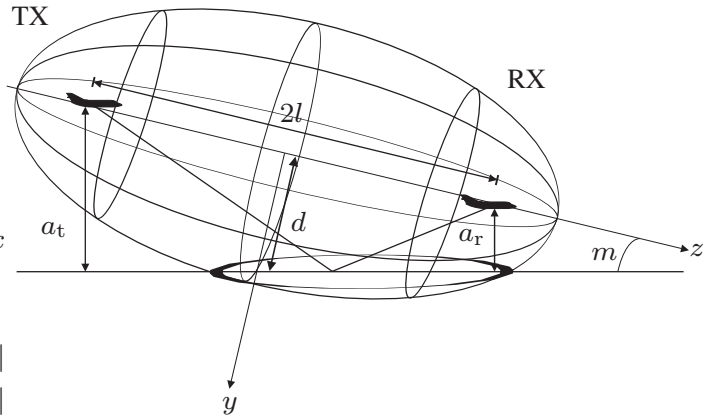

Fig. 1. Aircraft positions in the prolate spheroidal coordinate system. An ellipsoid represents the surface of constant $\xi$, which intersects with the ground forming an ellipse. The slope $m$ and the $y$-intercept $d$ of the ground intersecting the coordinate plane is also displayed.

\section{Prolate Spheroidal Coordinate System}

The mathematical tractability of the Doppler pdf, characteristic function, and limiting frequencies of a general 3D $\mathrm{M} 2 \mathrm{M}$ channel is based on the selection of an appropriate coordinate system. We use the A2A channel similar to [24] as an exemplary M2M channel. As shown in [32], a suitable coordinate system for the M2M channel is the PSCS, since it enables a natural delay-dependent description of the Doppler pdf. In general, the PSCS is a three-dimensional, curvilinear, orthogonal coordinate system, which adequately describes two-center problems, exemplified well by the A2A channel with the transmitter and receiver forming the two centers, as shown in Fig. 1. The origin of the coordinate system is always located in the middle between the two aircraft and moves along with them, which means the coordinate system itself becomes time-variant.

The relationship between Cartesian and prolate spheroidal coordinates is according to [35] given by

$$
\begin{aligned}
& x=l \sqrt{\left(\xi^{2}-1\right)\left(1-\eta^{2}\right)} \cos \vartheta, \\
& y=l \sqrt{\left(\xi^{2}-1\right)\left(1-\eta^{2}\right)} \sin \vartheta, \\
& z=l \xi \eta,
\end{aligned}
$$

with $l$ being the focus distance, i.e., the distance from the aircraft to the origin. The three coordinates are given by $\xi \in[1, \infty), \eta \in[-1,1]$ and $\vartheta \in[0,2 \pi)$. With this coordinate transform, the $z$-axis is always aligned with the major axis of the ellipsoid and the aircraft are found in the $y$-z-plane. Since the coordinate system is time-variant, the focus distance $l$ is time-variant as well. The coordinate $\xi=\frac{\tau}{\tau_{\text {los }}}$ corresponds to the propagation delay normalized by the LOS delay. This choice allows for a very general description of the Doppler frequency later, where the absolute delay between transmitter and receiver does not matter anymore. The absolute delay, however, can be calculated by $\tau=\tau_{\text {los }} \xi=\frac{2 l \xi}{c}$ with $c$ being the speed of light. Iso-surfaces of $\xi$ are prolate spheroidal ellipsoids, whereas constant values of $\eta$ produce hyperboloid surfaces. The third orthogonal surface in the PSCS is formed by a half-plane seen in Fig. 2. Geometrically, a fixed $\xi$ coordinate represents locations where the sum of the delays to the foci, i.e., aircraft, is constant. A fixed $\eta$-coordinate represents locations where the difference of the delays to the 


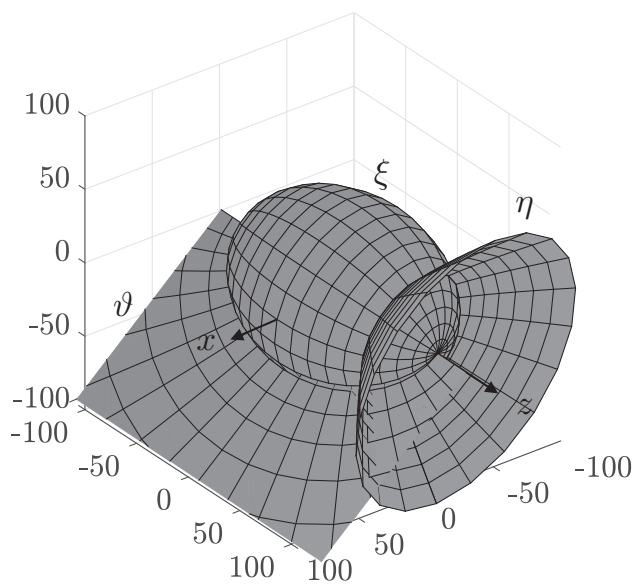

Fig. 2. Prolate spheroidal coordinate system with the surfaces of constant $\xi$ (ellipsoid), $\eta$ (hyperboloid), and $\vartheta$ (half-plane).

aircraft is constant, yet in contrast to $\xi$ we do not need to use this physical interpretation in the following. As we show later, this coordinate transform effectively leads to mathematically tractable expressions of the Doppler frequency.

Let us point out that the big advantage of the PSCS is that by fixing the $\xi$-coordinate, we naturally obtain a delaydependent description of the Doppler frequency. As such, the resulting description becomes only dependent on the other two coordinates $\eta$ and $\vartheta$ in the PSCS. In contrast to that, fixing the delay in a Cartesian coordinate system still implies the dependency on all three spatial coordinates $x$, $y$, and $z$, which makes the resulting expressions much more difficult to interpret and analyze. Thus, the description in the new coordinate system allows us to express both delay and Doppler frequency in mathematically tractable expressions in comparison to Cartesian coordinates.

The distance from transmitter to an arbitrary scatterer $d_{\mathrm{t}}$ and the distance from this scatterer to the transmitter $d_{\mathrm{r}}$ can be defined as

$$
\begin{aligned}
d_{\mathrm{t}} & =(\xi+\eta) l, \\
d_{\mathrm{r}} & =(\xi-\eta) l, \\
d_{\mathrm{sc}} & =d_{\mathrm{t}}+d_{\mathrm{r}}=2 \xi l,
\end{aligned}
$$

where the total distance $d_{\mathrm{sc}}$ or the total delay $\tau_{\mathrm{sc}}=\frac{d_{\mathrm{sc}}}{c}$ depends only on the $\xi$-coordinate. As has been shown by the authors in [23], the Doppler frequency is calculated as the spatial gradient of the transmitter and receiver distances $d_{\mathrm{t}}$ and $d_{\mathrm{r}}$ projected onto their velocity vectors, i.e.,

$$
f_{\mathrm{d}}(\mathbf{x})=\left(\mathbf{v}_{\mathrm{t}}^{\mathrm{T}} \nabla d_{\mathrm{t}}(\mathbf{x})+\mathbf{v}_{\mathrm{r}}^{\mathrm{T}} \nabla d_{\mathrm{r}}(\mathbf{x})\right) \frac{f_{\mathrm{c}}}{c} .
$$

A delay-dependent representation of the Doppler frequency in Cartesian coordinates depends, however, on the $x$-, $y$-, and $z$-coordinates, so that the calculation of the Doppler pdf becomes very cumbersome. To circumvent this, we transform the mathematical analysis in the PSCS and express the gradient in (3) in prolate spheroidal coordinates.
Consider an arbitrary scalar function $\Psi(\xi, \eta, \vartheta)$ in the PSCS. Its gradient $\nabla \Psi$ can be computed as [36]

$$
\nabla \Psi(\xi, \eta, \vartheta)=\frac{1}{h_{\xi}} \frac{\partial \Psi}{\partial \xi} \mathbf{e}_{\xi}+\frac{1}{h_{\eta}} \frac{\partial \Psi}{\partial \eta} \mathbf{e}_{\eta}+\frac{1}{h_{\vartheta}} \frac{\partial \Psi}{\partial \vartheta} \mathbf{e}_{\vartheta},
$$

where $\mathbf{e}_{\xi}, \mathbf{e}_{\eta}$, and $\mathbf{e}_{\vartheta}$ are the orthonormal basis vectors of the PSCS. They are obtained by transforming the Cartesian basis vectors $\mathbf{e}_{x}, \mathbf{e}_{y}$ and $\mathbf{e}_{z}$ using the following identity

$$
\mathbf{e}_{\alpha}=\frac{\partial x}{\partial \alpha} \frac{\mathbf{e}_{x}}{h_{\alpha}}+\frac{\partial y}{\partial \alpha} \frac{\mathbf{e}_{y}}{h_{\alpha}}+\frac{\partial z}{\partial \alpha} \frac{\mathbf{e}_{z}}{h_{\alpha}},
$$

where the subscript is in the set $\alpha \in\{\xi, \eta, \vartheta\}$. In (4) and (5) the scalars $h_{\xi}, h_{\eta}$, and $h_{\vartheta}$ are the so called scale factors. They account for the normalization of the basis vectors in the transformed coordinate system to ensure that the transformed basis is also orthonormal. For orthogonal coordinates the scale factors are the roots of the three non-zero elements $h_{i}=\sqrt{g_{i i}}$ of the metric tensor g, see [36] for more details. For the PSCS, the scale factors are calculated as

$$
\begin{aligned}
& h_{\xi}=l \sqrt{\frac{\xi^{2}-\eta^{2}}{\xi^{2}-1}}, \quad h_{\eta}=l \sqrt{\frac{\xi^{2}-\eta^{2}}{1-\eta^{2}}}, \\
& h_{\vartheta}=l \sqrt{\left(\xi^{2}-1\right)\left(1-\eta^{2}\right)} .
\end{aligned}
$$

By transforming (3) into prolate spheroidal coordinates, the Doppler frequency can be expressed as

$$
\begin{gathered}
f_{\mathrm{d}}(t ; \xi, \eta, \vartheta)=\frac{f_{\mathrm{c}}}{c}( \\
\frac{\sqrt{\left(\xi^{2}-1\right)\left(1-\eta^{2}\right)}}{\xi+\eta}\left(v_{\mathrm{t} x} \cos \vartheta+v_{\mathrm{t} y} \sin \vartheta\right)+\frac{\xi \eta+1}{\xi+\eta} v_{\mathrm{t} z} \\
\left.+\frac{\sqrt{\left(\xi^{2}-1\right)\left(1-\eta^{2}\right)}}{\xi-\eta}\left(v_{\mathrm{r} x} \cos \vartheta+v_{\mathrm{r} y} \sin \vartheta\right)+\frac{\xi \eta-1}{\xi-\eta} v_{\mathrm{r} z}\right),
\end{gathered}
$$

where $\mathbf{v}_{\mathrm{t}}=\left[v_{\mathrm{t} x}, v_{\mathrm{t} y} v_{\mathrm{t} z}\right]^{\mathrm{T}}$ and $\mathbf{v}_{\mathrm{t}}=\left[v_{\mathrm{r} x}, v_{\mathrm{r} y} v_{\mathrm{r} z}\right]^{\mathrm{T}}$ are the Cartesian components of the velocity vectors of TX and RX, respectively. The alignment of the PSCS and the Cartesian coordinate system is shown in Fig. 1. Due to the implicit time-variance of the velocity vectors, the Doppler frequency becomes time-variant, which is noted by the variable $t$. In the following, we show how delay-dependent Doppler pdfs and the corresponding characteristic functions are computed for A2A channels in the PSCS.

\section{A. Time-Variant, Delay-Dependent Doppler PDF}

In an A2A scenario, the scattering of the transmitted signal takes only place on the ground. Thus, we expect the scattering to take place when the propagation delay exceeds a certain value. Geometrically, this corresponds to the delay ellipsoid intersecting the ground plane, see also Fig. 1. Naturally, this intersection results in an ellipse - the scattering ellipse. A plane ground obviously is an approximation of the curved earth for relatively small distances and low altitudes. This assumption is, however, fulfilled for low altitudes or take-off and landing scenarios, since the power of the scattering from the ground is low and thus only received at low altitudes. At cruising altitude the received scattering is negligible. 
In the following, we study the delay-dependent conditional Doppler pdf $p\left(t ; f_{\mathrm{d}} \mid \xi\right)$ in the PSCS that is induced by the scatterers lying on the scattering ellipse. The derivation is obtained by a coordinate transformation of the scatterer distribution $p(\eta \mid \xi)$ as has been shown in [32]. In this paper, however, we extend the 2D V2V case from [32] to a 3D A2A configuration. To this end, we will summarize the key steps in computing $p\left(t ; f_{\mathrm{d}} \mid \xi\right)$ for this set-up.

For this reason, we assume that the scatterers are uniformly distributed on the scattering ellipse. As such, the distribution of the scatterers will be inversely proportional to the length of the scattering ellipse. To compute the length of the ellipse in the PSCS, we recall that a general differential element for the $3 \mathrm{D}$ case can be computed as follows

$$
\mathrm{d} s=\sqrt{h_{\xi}^{2} \mathrm{~d} \xi^{2}+h_{\eta}^{2} \mathrm{~d} \eta^{2}+h_{\vartheta}^{2} \mathrm{~d} \vartheta^{2}} .
$$

Now we distinguish two cases for aircraft arrangement. In Case 1 we consider a situation, in which two aircraft are flying directly above each other so that $z$-direction is normal to the ground plane. In Case 2 we consider all other remaining configurations.

Case 1: Since the aircraft are vertically above each other, the scattering ellipse becomes a circle for $2 l \xi>a_{\mathrm{t}}+a_{\mathrm{r}}$. The latter condition implies that the delay ellipsoid is large enough to intersect the ground plane. For this case there exists a fixed relationship between coordinates $\xi$ and $\eta$ given by

$$
\eta=\left\{\begin{array}{rc}
\frac{a_{\mathrm{t}}+a_{\mathrm{r}}}{2 l \xi}, & a_{\mathrm{t}}>a_{\mathrm{r}} \\
-\frac{a_{\mathrm{t}}+a_{\mathrm{r}}}{2 l \xi}, & a_{\mathrm{t}}<a_{\mathrm{r}} .
\end{array}\right.
$$

Now, we insert (9) into (7) to eliminate the dependency on $\eta$ in the Doppler frequency. Therefore, the Doppler frequency only depends on $\vartheta$, since $\xi$ is fixed. The differential length in (8) can be computed as

$$
\mathrm{d} s=h_{\vartheta} \mathrm{d} \vartheta=l \sqrt{\left(\xi^{2}-1\right)\left(1-\eta^{2}\right)} \mathrm{d} \vartheta,
$$

since $\mathrm{d} \xi=\mathrm{d} \eta=0$. Thus, $p\left(t ; f_{\mathrm{d}} \mid \xi\right)$ is a transformation of the angular distribution $p(\vartheta \mid \xi)$. The latter can be computed for Case 1 as

$$
p(\vartheta \mid \xi)=\frac{l \sqrt{\left(\xi^{2}-1\right)\left(1-\eta^{2}\right)}}{\int_{0}^{2 \pi} l \sqrt{\left(\xi^{2}-1\right)\left(1-\eta^{2}\right)} \mathrm{d} \vartheta}=\frac{1}{2 \pi} .
$$

This result shows that the distribution of the scatterers on the circle is uniform. Moreover, such a distribution automatically induces a Doppler spectrum that coincides with the wellknown Jakes spectrum [37], which can be created by equivalent scatterers on a circle.

For the time-variant, delay-dependent Doppler frequency pdf, we then get the following expression

$$
\begin{aligned}
p\left(t ; f_{\mathrm{d}} \mid \xi\right) & =\left.\sum_{\vartheta^{\prime} \in\left\{\mathcal{F}-1\left(f_{\mathrm{d}}\right)\right\}} \frac{p(\vartheta \mid \xi)}{\left|\frac{\partial f_{\mathrm{d}}(t ; \vartheta \mid \xi)}{\partial \vartheta}\right|}\right|_{\vartheta=\vartheta^{\prime}} \\
& =\left.\sum_{\vartheta^{\prime} \in\left\{\mathcal{F}-1\left(f_{\mathrm{d}}\right)\right\}} \frac{1}{2 \pi\left|\frac{\partial f_{\mathrm{d}}(t ; \vartheta \mid \xi)}{\partial \vartheta}\right|}\right|_{\vartheta=\vartheta^{\prime}},
\end{aligned}
$$

with $\vartheta \in[0,2 \pi)$. The sum in (12) accounts for the fact that one Doppler frequency results possibly in several values of $\vartheta$. Due to the trigonometric functions, there are at most two values of $\vartheta$ with the same Doppler frequency, since the Doppler frequency is periodic with $2 \pi$.

Case 2: Here, the intersection of the delay ellipse with the ground plane is no longer a circle, but rather an ellipse. In this case, the relationship between $\eta$ and $\vartheta$ can be established through an analysis of the following set of equations

$$
\begin{aligned}
y & =m z+d \\
l \sqrt{\left(\xi^{2}-1\right)\left(1-\eta^{2}\right)} \sin \vartheta & =m l \xi \eta+d,
\end{aligned}
$$

where $m$ is the slope of the ground plane with respect to the major axis of the ellipsoid and $d$ the intercept of the $y$-axis with the ground plane as shown in Fig. 1. The slope $m$ is calculated as

$$
m=\frac{a_{\mathrm{r}}-a_{\mathrm{t}}}{\sqrt{(2 l)^{2}-\left(a_{\mathrm{r}}-a_{\mathrm{t}}\right)^{2}}},
$$

and the intercept $d$ of the ground plane with the $y$-axis is computed from the relationship

$$
d=\sqrt{m^{2}+1} a_{\mathrm{t}}+m l .
$$

In the $\mathrm{A} 2 \mathrm{~A}$ case the parameter $\eta$ in general does not attain the whole range $\eta \in[-1,1]$. It is only attainable, when the scattering ellipse lies in the $x$-z-plane, i.e., both aircraft are on the ground. To find the attainable range of $\eta$ values in a particular aircraft configuration, we solve (13) for $\eta$ with $\sin (\vartheta)= \pm 1$ in order to calculate the maximum $\eta_{\max }$ and minimum $\eta_{\min }$ values of $\eta$ as

$$
\begin{aligned}
\eta_{\min } & =-\frac{d m \xi-\sqrt{\left(\xi^{2}-1\right)\left(l^{2}\left(\left(1+m^{2}\right) \xi^{2}-1\right)-d^{2}\right)}}{l\left(\left(1+m^{2}\right) \xi^{2}-1\right)}, \\
\eta_{\max } & =-\frac{d m \xi+\sqrt{\left(\xi^{2}-1\right)\left(l^{2}\left(\left(1+m^{2}\right) \xi^{2}-1\right)-d^{2}\right)}}{l\left(\left(1+m^{2}\right) \xi^{2}-1\right)} .
\end{aligned}
$$

The fixed relation between $\eta$ and the angle $\vartheta$ for the resulting scattering ellipse on the ground can then be established again from (13), which gives the following relationship

$$
\vartheta=\arcsin \left(\frac{m l \xi \eta+d}{l \sqrt{\left(\xi^{2}-1\right)\left(1-\eta^{2}\right)}}\right) .
$$

Finally, we insert (17) into (7) to eliminate $\vartheta$. By doing this, we eliminate the dependency on the trigonometric functions in (7) and define

$$
w=\frac{m l \xi \eta+d}{l \sqrt{\left(\xi^{2}-1\right)\left(1-\eta^{2}\right)}}
$$

to simplify the further notation.

Let us point out that the principal value of the arcsin function is defined for values $[-\pi / 2, \pi / 2]$. It follows then that in (17) we have to distinguish the left and right half-space $\mathcal{S}_{1}=\{\vartheta: \vartheta \in[-\pi / 2, \pi / 2)\}$ and $\mathcal{S}_{2}=\{\vartheta: \vartheta \in[\pi / 2,3 \pi / 2)\}$ to cover the complete range of $2 \pi$. In one half-space, the $x$ component of the velocity vectors have a positive sign and in the other half-space a negative sign. After combining these half-spaces together and inserting (17) in (7) we obtain the 
expression for the Doppler frequency in Case 2 without any trigonometric functions as follows

$$
\begin{aligned}
& f_{\mathrm{d} \pm}(t ; \xi, \eta)=\frac{f_{\mathrm{c}}}{c}\left(\frac{\xi \eta+1}{\xi+\eta} v_{\mathrm{t} z}+\frac{\xi \eta-1}{\xi-\eta} v_{\mathrm{r} z}\right. \\
& +\frac{\sqrt{\left(\xi^{2}-1\right)\left(1-\eta^{2}\right)}}{\xi+\eta}\left( \pm v_{\mathrm{t} x} \sqrt{1-w^{2}}+v_{\mathrm{t} y} w\right) \\
& \left.+\frac{\sqrt{\left(\xi^{2}-1\right)\left(1-\eta^{2}\right)}}{\xi-\eta}\left( \pm v_{\mathrm{r} x} \sqrt{1-w^{2}}+v_{\mathrm{r} y} w\right)\right)
\end{aligned}
$$

The Doppler frequency is split up in two parts for the two half-planes: $f_{\mathrm{d}+}$, where all terms in (19) have positive leading signs, and $f_{\mathrm{d}-}$, where the leading sign in front of $v_{\mathrm{t} x}$ and $v_{\mathrm{r} x}$ is negative.

Let us consider a differential line element on the ellipsoidal surface, i.e., when $\xi$ is fixed. In this case (8) can be formulated as

$$
\mathrm{d} s=l \sqrt{\frac{\xi^{2}-\eta^{2}}{1-\eta^{2}}+\left(\xi^{2}-1\right)\left(1-\eta^{2}\right)\left(\frac{\mathrm{d} \vartheta}{\mathrm{d} \eta}\right)^{2}} \mathrm{~d} \eta
$$

where $\mathrm{d} \xi=0$ on the ellipsoidal surface. We restrict the ellipsoid surface to a scattering ellipse on the ground. This restriction invokes the fixed relation between coordinates $\eta$ and $\vartheta$ in (17), which eventually allows us to express the differential length segment as a function of the coordinate $\eta$ only.

Consider the parameter $\eta \in\left[\eta_{\min }, \eta_{\max }\right]$ of the half-ellipse that specifies a scatterer lying on it. For a fixed $\xi$, it can be shown that the conditional pdf $p(\eta \mid \xi)$ can be computed in each half-space $\mathcal{S}_{1}$ and $\mathcal{S}_{2}$ by applying standard rules of probability density transformations [38] by

$$
\begin{aligned}
p(\eta \mid \xi) & =\left.\frac{1}{2} p_{1}(\eta \mid \xi)\right|_{\vartheta(\eta) \in \mathcal{S}_{1}}+\left.\frac{1}{2} p_{2}(\eta \mid \xi)\right|_{\vartheta(\eta) \in \mathcal{S}_{2}} \\
& =\frac{1}{2} \frac{l \sqrt{\frac{\xi^{2}-\eta^{2}}{1-\eta^{2}}+\left(\xi^{2}-1\right)\left(1-\eta^{2}\right)\left(\frac{\mathrm{d} \vartheta}{\mathrm{d} \eta}\right)^{2}}}{\int_{\eta_{\min }}^{\eta_{\max }} l \sqrt{\frac{\xi^{2}-\eta^{2}}{1-\eta^{2}}+\left(\xi^{2}-1\right)\left(1-\eta^{2}\right)\left(\frac{\mathrm{d} \vartheta}{\mathrm{d} \eta}\right)^{2}} \mathrm{~d} \eta} \\
& +\frac{1}{2} \frac{l \sqrt{\frac{\xi^{2}-\eta^{2}}{1-\eta^{2}}+\left(\xi^{2}-1\right)\left(1-\eta^{2}\right)\left(\frac{\mathrm{d} \vartheta}{\mathrm{d} \eta}\right)^{2}}}{\int_{\eta_{\min }}^{\eta_{\max }} l \sqrt{\frac{\xi^{2}-\eta^{2}}{1-\eta^{2}}+\left(\xi^{2}-1\right)\left(1-\eta^{2}\right)\left(\frac{\mathrm{d} \vartheta}{\mathrm{d} \eta}\right)^{2}} \mathrm{~d} \eta}
\end{aligned}
$$

where $\frac{\mathrm{d} \vartheta}{\mathrm{d} \eta}$ is calculated using (17). The pdf $p(\eta \mid \xi)$ could be simplified, since the probability in both half-spaces is naturally the same. Yet, we purposely keep the expression in this form, since the Doppler frequency can be different in both halfplanes.

Following [32], we compute a time-variant, delay-dependent Doppler pdf as

$$
\begin{aligned}
p\left(t ; f_{\mathrm{d}} \mid \xi\right) & =\left.\sum_{\eta^{\prime} \in\left\{\mathcal{F}^{-1}\left(f_{\mathrm{d}+}\right)\right\}} \frac{1}{2} \frac{p_{1}(\eta \mid \xi)}{\left|\frac{\partial f_{\mathrm{d}+}(t ; \eta \mid \xi)}{\partial \eta}\right|}\right|_{\eta=\eta^{\prime}} \\
& +\left.\sum_{\eta^{\prime} \in\left\{\mathcal{F}^{-1}\left(f_{\mathrm{d}-}\right)\right\}} \frac{1}{2} \frac{p_{2}(\eta \mid \xi)}{\left|\frac{\partial f_{\mathrm{d}-}(t ; \eta \mid \xi)}{\partial \eta}\right|}\right|_{\eta=\eta^{\prime}},
\end{aligned}
$$

with the Doppler frequency $f_{\mathrm{d} \pm}(t ; \xi, \eta)$ computed according to (19). The sum in (22) accounts for the fact that a single Doppler frequency value can correspond possibly to several values of $\eta$. However, the maximum number of possible values for $\eta$ leading to the same Doppler frequency is four, which we prove later here using the theory of algebraic curves.

In [33] we have shown the proportionality between the Doppler power spectrum and the Doppler pdf for the US case. Using the properties of the Fourier transform, this proportionality translates into the proportionality between the correlation function and the corresponding characteristic function. The latter becomes a time-variant and delay-dependent function that has the same interpretation as the correlation function yet in a non-stationary setting.

\section{B. Time-Variant, Delay-Dependent Characteristic Function}

The characteristic function is defined as the inverse Fourier transform of the probability density function, see, e.g., [38] and [39]. It thus gives an alternative description of a random variable and can be used to facilitate the computation of the moments of a random variable, or compute a distribution of a sum of independent random variables.

In order to calculate the characteristic function, we compute the inverse Fourier transform of the pdf $p_{i}(\eta \mid \xi)$ with $i=1,2$ instead of using the Doppler pdf $p\left(t ; f_{\mathrm{d}} \mid \xi\right)$ for both half-spaces $\mathcal{S}_{1}$ and $\mathcal{S}_{2}$. We show the calculations only for Case 2; for Case 1 the computation can be performed in a similar way with the distinction that instead of using $p_{1}(\eta \mid \xi)$ and $p_{2}(\eta \mid \xi)$, the scatterer distribution $p(\vartheta \mid \xi)$ is used. A similar approach of calculating the characteristic function is presented in [39, Appendix A]. Thus the characteristic function is calculated as

$$
\begin{aligned}
\Phi(t ; u \mid \xi) & =\frac{1}{2} \int_{\eta_{\min }}^{\eta_{\max }} p_{1}(\eta \mid \xi) \exp \left(\mathrm{j} 2 \pi u f_{\mathrm{d}+}(t ; \xi, \eta)\right) \mathrm{d} \eta \\
& +\frac{1}{2} \int_{\eta_{\min }}^{\eta_{\max }} p_{2}(\eta \mid \xi) \exp \left(\mathrm{j} 2 \pi u f_{\mathrm{d}-}(t ; \xi, \eta)\right) \mathrm{d} \eta
\end{aligned}
$$

where $f_{\mathrm{d}+}$ is the Doppler frequency in the half-space $\mathcal{S}_{1}$ and $f_{\mathrm{d}-}$ the Doppler frequency in the half-space $\mathcal{S}_{2}$ according to (19). The variable $u:=\Delta t-$ an independent variable of the characteristic function - is equivalent to a time lag in the classical correlation function.

The characteristic function - an equivalent representation of the channel correlation function - permits deriving other important statistical parameters that summarize the instantaneous dynamics of the channel. Thus, we can determine the time-variant, delay-dependent, first and second central moments of the channel Doppler spread, which are known as the mean Doppler $\mu(t ; \xi)$ and the corresponding standard deviation $\sigma(t ; \xi)$. This is done by calculating the first and second derivative of the characteristic function at $u=0$. 
The first and second derivative with respect to the coordinate $u$ of the characteristic function $\Phi(t ; u \mid \xi)$ are calculated to determine the mean and Doppler spread like in [39] as

$$
\begin{aligned}
& \mu(t ; \xi)=\left.\frac{\frac{\partial}{\partial u} \Phi(t ; u \mid \xi)}{\mathrm{j} 2 \pi}\right|_{u=0}, \\
& \sigma(t ; \xi)=\left.\frac{\sqrt{\left(\frac{\partial}{\partial u} \Phi(t ; u \mid \xi)\right)^{2}-\frac{\partial^{2}}{\partial u^{2}} \Phi(t ; u \mid \xi)}}{2 \pi}\right|_{u=0} .
\end{aligned}
$$

The first derivative $\frac{\partial}{\partial u} \Phi(t ; u \mid \xi)$ can be computed with the following expression

$$
\begin{aligned}
& \frac{\partial}{\partial u} \Phi(t ; u \mid \xi)=\mathrm{j} 2 \pi( \\
& \frac{1}{2} \int_{\eta_{\min }}^{\eta_{\max }} p_{1}(\eta \mid \xi) f_{\mathrm{d}+}(t ; \xi, \eta) \exp \left(\mathrm{j} 2 \pi u f_{\mathrm{d}+}(t ; \xi, \eta)\right) \mathrm{d} \eta \\
& \left.+\frac{1}{2} \int_{\eta_{\min }}^{\eta_{\max }} p_{2}(\eta \mid \xi) f_{\mathrm{d}-}(t ; \xi, \eta) \exp \left(\mathrm{j} 2 \pi u f_{\mathrm{d}-}(t ; \xi, \eta)\right) \mathrm{d} \eta\right),
\end{aligned}
$$

and the second derivative $\frac{\partial^{2}}{\partial u^{2}} \Phi(t ; u \mid \xi)$ is similarly given as

$$
\begin{gathered}
\frac{\partial^{2}}{\partial u^{2}} \Phi(t ; u \mid \xi)=-4 \pi^{2}( \\
\frac{1}{2} \int_{\eta_{\min }}^{\eta_{\max }} p_{1}(\eta \mid \xi) f_{\mathrm{d}+}(t ; \xi, \eta)^{2} \exp \left(\mathrm{j} 2 \pi u f_{\mathrm{d}+}(t ; \xi, \eta)\right) \mathrm{d} \eta \\
\left.+\frac{1}{2} \int_{\eta_{\min }}^{\eta_{\max }} p_{2}(\eta \mid \xi) f_{\mathrm{d}-}(t ; \xi, \eta)^{2} \exp \left(\mathrm{j} 2 \pi u f_{\mathrm{d}-}(t ; \xi, \eta)\right) \mathrm{d} \eta\right) .
\end{gathered}
$$

By using $\frac{\partial^{2}}{\partial u^{2}} \Phi(t ; u \mid \xi)$, it becomes possible to calculate the delay-dependent level crossing rate (LCR) and average duration of fades (ADF), if Rayleigh or Rice fading for the amplitude distribution is assumed [39].

\section{Algebraic Analysis of the Doppler PDF of the Aeronautical Channel}

In what follows, we perform a detailed analysis of the delaydependent Doppler spectrum computed in the previous sections, specifically for the A2A case. Our intention is to study the poles of $p\left(t ; f_{\mathrm{d}} \mid \xi\right)$ caused by the extrema of the Doppler frequency for arbitrary delays. These constitute themselves as the zeros of $\partial f_{\mathrm{d}}(t ; \eta, \vartheta, \xi) / \partial \vartheta$ for Case 1 or $\partial f_{\mathrm{d}}(t ; \eta, \vartheta, \xi) / \partial \eta$ for Case 2. The set of extrema naturally include minimum and maximum Doppler frequencies, since due to the physical limitation of the Doppler frequency $f_{\mathrm{d}}$, the corresponding pdf $p\left(t ; f_{\mathrm{d}} \mid \xi\right)$ must have a finite support. For Case 1, we obtain

$$
\begin{aligned}
& \frac{\partial f_{\mathrm{d}}(t ; \xi, \eta, \vartheta)}{\vartheta}= \\
& \frac{f_{\mathrm{c}}}{c}\left(\frac{\sqrt{\left(\xi^{2}-1\right)\left(1-\eta^{2}\right)}}{\xi+\eta}\left(v_{\mathrm{t} y} \cos \vartheta-v_{\mathrm{t} x} \sin \vartheta\right)\right. \\
& \left.+\frac{\sqrt{\left(\xi^{2}-1\right)\left(1-\eta^{2}\right)}}{\xi-\eta}\left(v_{\mathrm{r} y} \cos \vartheta-v_{\mathrm{r} x} \sin \vartheta\right)\right),
\end{aligned}
$$

with two solutions for $\vartheta \in[0,2 \pi)$.

Now, we look at the more interesting Case 2. To this end, we use the theory of algebraic curves [40] and consider the curve $\mathcal{C}\left(f_{\mathrm{d}}, \eta\right)$ in coordinates $f_{\mathrm{d}}$ and $\eta$. The curve is obtained by isolating the roots in (19) and squaring the resulting equation. This leads to an algebraic curve of degree six with the highest monomial $f_{\mathrm{d}}^{2} \eta^{4}$. The highest exponent of variable $\eta$ is four, which means the resulting curve can have a maximum of four solutions of (31) for a fixed Doppler frequency $f_{\mathrm{d}}$ according to Bézout's theorem [41]. The curve $\mathcal{C}\left(f_{\mathrm{d}}, \eta\right)$ is given by the following expression in (31).

To be able to determine the extrema of the Doppler frequency let us consider the gradient $\left[\partial \mathcal{C} / \partial \eta, \partial \mathcal{C} / \partial f_{\mathrm{d}}\right]^{\mathrm{T}}$ of the algebraic curve. The extrema of the Doppler frequency are obtained as points on the curve that satisfy $\partial \mathcal{C} / \partial \eta=0$ and $\partial \mathcal{C} / \partial f_{\mathrm{d}} \neq 0$, i.e., these are the points, where the gradient is normal to the $\eta$-axis. Clearly, we see from (22) that these extrema will induce poles in the resulting Doppler spectrum. After some involved but straightforward algebra, these poles can be computed as roots of the polynomial $g(\eta)$

$$
g(\eta)=a_{6} \eta^{6}+a_{5} \eta^{5}+a_{4} \eta^{4}+a_{3} \eta^{3}+a_{2} \eta^{2}+a_{1} \eta+a_{0} .
$$

Let us point out that coefficients $a_{i}, i=0, \ldots, 6$, are represented by rather lengthy expressions, which, nonetheless, can be computed in closed form.Using (29) it becomes possible to bound the total number of poles in the Doppler spectrum. Despite the polynomial in (29) having a rather involved form of the coefficients, it structurally coincides with the polynomial obtained in the V2V case in [34], i.e., it has the same order.

By solving (29), we are able to calculate the delaydependent minimum and maximum Doppler frequency for any position and velocity vector configuration of the A2A channel. A general formula for solving solvable sextic equations is provided in [42].

In addition to the roots of (29) that indicate locations on the curve with the gradient normal to the $\eta$-axis, $\mathcal{C}\left(\eta, f_{\mathrm{d}}\right)$ in $(31)$ possesses singular points at locations where $\left[\partial \mathcal{C} / \partial f_{\mathrm{d}}, \partial \mathcal{C} / \partial \eta\right]=0$. For the considered case the location of the singular point can be computed in closed form as follows

$$
\eta_{\mathrm{s}}=\frac{v_{\mathrm{t} x}+v_{\mathrm{r} x}}{v_{\mathrm{t} x}-v_{\mathrm{r} x}} \xi .
$$

One might inquire, if the singular point can also contribute to the extrema of the Doppler frequency. Unfortunately, a formal answer to this question is elusive at the moment. Yet, our experimental evidence allows us to state certain conjectures about the behavior of the singular point and the zeros of (29).

First, a closer look at (30) reveals that this singular point is only observable, if $v_{\mathrm{t} x}+v_{\mathrm{r} x}<v_{\mathrm{t} x}-v_{\mathrm{r} x}$, since $|\eta| \leq 1$ and $\xi>1$ for scattering by definition. This means that the velocity vectors in the $x$-direction have to be anti-parallel. Moreover, $\eta_{\mathrm{s}}$ is likely to be present for small $\xi$ under realistic velocity assumptions. We also observed in [34] that the maximum number of poles is likewise typically observed for small delays. For $\xi \rightarrow 1$ the poles of (29) can be computed in closed form. Two of them are given for $\eta \rightarrow \frac{v_{t x}-v_{\mathrm{r} x}}{v_{\mathrm{tx}}+v_{\mathrm{rx}}}$, which is exactly the reverse condition for the existence of the singular point, see also Sec. III-A later. This allows us to state the following. 


$$
\begin{aligned}
& \left(f_{\mathrm{d}} \frac{c}{f_{\mathrm{c}}}\left(\xi^{2}-\eta^{2}\right)-(\xi \eta+1)(\xi-\eta) v_{\mathrm{t} z}-(\xi \eta-1)(\xi+\eta) v_{\mathrm{r} z}-\sqrt{\left(\xi^{2}-1\right)\left(1-\eta^{2}\right)}\left(v_{\mathrm{t} y} w(\xi-\eta)+v_{\mathrm{r} y} w(\xi+\eta)\right)\right)^{2}= \\
& \left( \pm \sqrt{\left(\xi^{2}-1\right)\left(1-\eta^{2}\right)}\left(v_{\mathrm{t} x} \sqrt{1-w^{2}}(\xi-\eta)+v_{\mathrm{r} x} \sqrt{1-w^{2}}(\xi-\eta)\right)\right)^{2}
\end{aligned}
$$

Conjecture 1. The number of real roots given by the polynomial (29) is 6 , if the $x$-components of the velocity vectors point in the same direction, i.e., when $v_{\mathrm{t} x}+v_{\mathrm{r} x}>v_{\mathrm{t} x}-v_{\mathrm{r} x}$. If the velocity vectors in the $x$-direction are anti-parallel, (29) has a maximum of 4 real roots and a singular point according to (30) is observed.

This means that the total number of poles in the Doppler spectrum remains at 6 according to our theorem in [34], although the number of solutions for $\partial \mathcal{C} / \partial \eta=0$ increase to 7 to account for the singular point. As such, Theorem 1 in [34] likewise applies here and holds in general for A2A channels. Thus, the Doppler spectrum for general A2A channels is also characterized by at most 6 poles. In contrast to the $\mathrm{V} 2 \mathrm{~V}$ channel, though, the domain of $\eta$ in the A2A case is restricted to the interval $\left[\eta_{\min }, \eta_{\max }\right]$ given in (16).

Now, we return to the singular point $\eta_{s}$ and study it in more detail. In general, the singular point can be classified as a so called crunode, acnode or cusp on the curve. See [40] for more information. The type of singular point is determined from the properties of the Hessian matrix evaluated at the singular point on the curve [40]. In particular, for

$$
\left(\frac{\partial^{2} \mathcal{C}}{\partial f_{\mathrm{d}} \partial \eta}\right)^{2}>,<, \text { or }=\frac{\partial^{2} \mathcal{C}}{\partial f_{\mathrm{d}}^{2}} \frac{\partial^{2} \mathcal{C}}{\partial \eta^{2}}
$$

we obtain a crunode, an acnode, or a cusp, respectively.

Let us discuss the nature of a singular point in the context of channel modeling by providing some underlying physical interpretation. In general, the singular point is obtained when the curve $\mathcal{C}$ crosses itself in a plane vertical to the $y$ - $z$ plane, i.e., in the ground plane here. Depending on the spatial relationship between the aircraft and the ground plane, we get the following properties:

- A crunode is obtained when the crossing point lies within the intersection plane, i.e, for $\eta_{\min }<\eta<\eta_{\max }$,

- A cusp is obtained when the crossing point is at the border of the intersection plane, i.e, for $\eta \in\left\{\eta_{\min }, \eta_{\max }\right\}$, and finally,

- An acnode is obtained when the crossing point is found outside the intersection plane, i.e., for $-1 \leq \eta<\eta_{\min }$ or $\eta_{\max }<\eta \leq 1$.

Expression (30) naturally holds for V2V channels as well. However, in 2D V2V channels, like those considered in [33], acnodes do not occur, since $-1 \leq \eta \leq 1$, i.e., the crossing point always lies within the intersection plane. The different types of singular points are visualized in Fig. 3 for different delays $\xi$. To generate these curves we used Scenario 4 that will be discussed later in Section IV. In Table I, we summarize types of a singular points for the delay range $1<\xi \leq 9$.
TABLE I

GENERAL CLASSIFICATION OF SINGULAR POINTS FOR SCENARIO 4

\begin{tabular}{|c|c|c|c|} 
Normalized Delay & Crunode & Cusp & Acnode \\
\hline $1<\xi<1.422$ & & & $\mathrm{o}$ \\
\hline$\xi=1.422$ & & $\diamond$ & \\
\hline $1.422<\xi<7.987$ & $\mathrm{x}$ & & \\
\hline$\xi=7.987$ & & $\diamond$ & \\
\hline $7.987<\xi<=9$ & & & $\mathrm{o}$ \\
\hline
\end{tabular}

TABLE II

SPECIFIC VALUES FOR THE SINGULAR POINTS SHOWN IN FIG. 3

\begin{tabular}{|c|c|c|c|}
$\xi$ & $\eta$ & $f_{\mathrm{d}}$ & Singular Point \\
\hline 1.3 & -0.144 & $-79.27 \mathrm{~Hz}$ & Acnode \\
\hline 1.422 & -0.158 & $-76.32 \mathrm{~Hz}$ & Cusp \\
\hline 1.707 & -0.189 & $-73.03 \mathrm{~Hz}$ & Crunode \\
\hline 2.943 & -0.327 & $-73.56 \mathrm{~Hz}$ & Crunode \\
\hline 7.987 & -0.878 & $-138.08 \mathrm{~Hz}$ & Cusp \\
\hline 8.5 & -0.944 & $-147.19 \mathrm{~Hz}$ & Acnode \\
\hline
\end{tabular}

As we can see, for $1<\xi<1.422$ an acnode is obtained. For $\xi \geq 1.247$ the Doppler curve $\mathcal{C}$ begins to intersect the ground plane. The blue curve in Fig. 3 shows the result for $\xi=1.3$. As we can see, the curve is closed, possesses two extreme Doppler frequencies, and has one acnode. As the delay reaches $\xi=1.422$, a cusp is obtained. Still, for $\xi=1.422$ we obtain two extreme Doppler frequencies.

As the delay increases, the singular point then becomes a crunode for $1.422<\xi<7.987$. This implies that the curve $\mathcal{C}$ crosses itself once in the ground plane. We can see this in Fig. 3 for $\xi=1.707$. Here, four extreme Doppler frequencies are obtained. For $\xi=2.943$, however, the curve still has the crunode, yet the number of Doppler extrema reduces to only two. For $\xi=7.987$ the crunode becomes a cusp again and then it changes to an acnode for $7.987<\xi<=9$. An example for the acnode at $\xi=8.5$ is shown in Fig. 3 .

Let us mention that singular points are observable due to the fact that the Doppler frequency representation in (7) is squared in (29). In our previous work [34], the analysis of the Doppler frequency has been done by distinguishing solutions in the different $\vartheta$-half-planes $\mathcal{S}_{1}$ and $\mathcal{S}_{2}$. Since these cases were considered separately, the singular points were thus not observed. The approach used here, however, reveals these singular points and in this respect generalizes the result in [34].

Finally, we display the values of the real roots of the polynomial and the singular point (green line) in Fig 4 . Interestingly, the real roots start in pairs of two for small, but different delays. As the delay reaches $\xi=1.247$, the ellipsoid intersects the ground plane for the first time. At this delay, the roots marked by the blue and red line appear. The second pair 


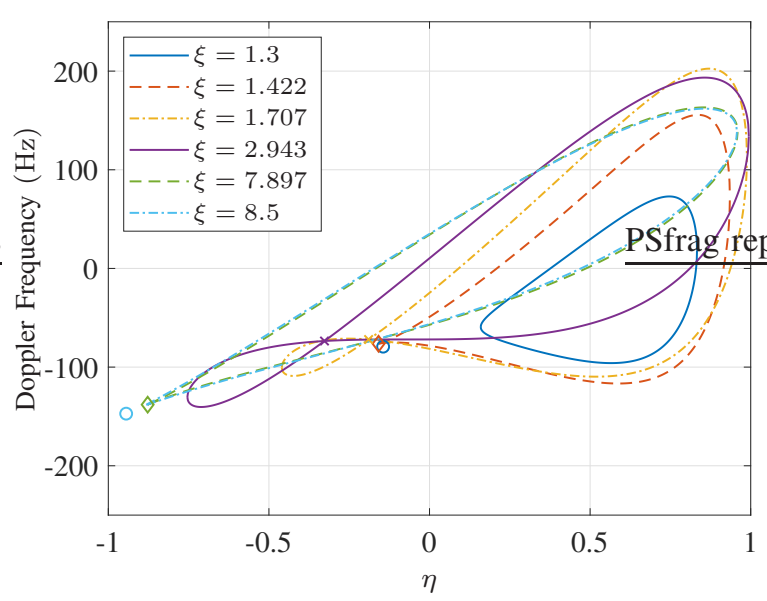

Fig. 3. Sextic curve $\mathcal{C}\left(f_{\mathrm{d}}, \eta\right)$ for Scenario 4. The singular points are acnodes, cusps, and crunodes, which are marked as circles, diamonds, and crosses, respectively.

of real roots, marked by the yellow and purple lines, appears as the delay reaches $\xi=1.4$. The roots represented by the red and yellow curves become a double root at the delay $\xi=2.94$; yet as the delay increases further they become a complex conjugated pair of roots and they disappear from the spectrum. Thus, only one root pair remains, which then leads to the minimum and maximum Doppler frequencies.

The classification of the singular point can also be derived from Fig 4. In particular, the intersections of the green line, i.e., the locations of the singular point $\eta_{s}$, with the locations of the roots $\eta_{3}$ (orange line) and $\eta_{4}$ (purple line) are of interest. Although the exact numerical analysis of the intersections is quite challenging, the empirical analysis of the results allows us to state the following: In case of a cusp, the intersection of the singular point with the root implies that the resulting point is not necessarily an extreme point with $\partial f_{\mathrm{d}} / \partial \eta=0$; rather it defines the boundary value of the Doppler frequency given by the $\eta_{\min }$ (or $\eta_{\max }$ ) curve. This can be seen for $\xi=1.422$ and $\xi=8.5$, where $\eta_{4}=\eta_{\mathrm{s}}=\eta_{\min }$.

In case of a crunode the intersection leads to an extreme point in one of the branches of the curve $\mathcal{C}$, where $\eta_{3}=\eta_{\mathrm{s}}$. This can be well seen at $\xi=1.510$ and $\xi=2.524$, where the green curve intersects the yellow curve in Fig 4.

The singular point given by (30) is valid for all intersection planes that are orthogonal to the $y-z$-plane; this naturally includes any ground plane. Moreover, in this case only the velocities in the $x$-direction determine the location of the singular point. A similar argument can be used for vertical intersection planes, such as building walls in street canyons, since these are the intersection planes orthogonal to the $x-z$ plane. Thus, only velocities in the $y$-direction are responsible for the emergence of the singular points. An example of this case is, however, left outside the scope of this work as it is less relevant for aircraft.

Finally, we consider the following degenerate case: The velocity vector components are $v_{\mathrm{t} x}=v_{\mathrm{r} x}=v_{\mathrm{t} y}=v_{\mathrm{r} y}=0$ and the aircraft are at the same altitude, i.e., $a_{\mathrm{t}}=a_{\mathrm{r}}$. In general for degenerate cases, roots of the polynomial $g(\eta)$ in

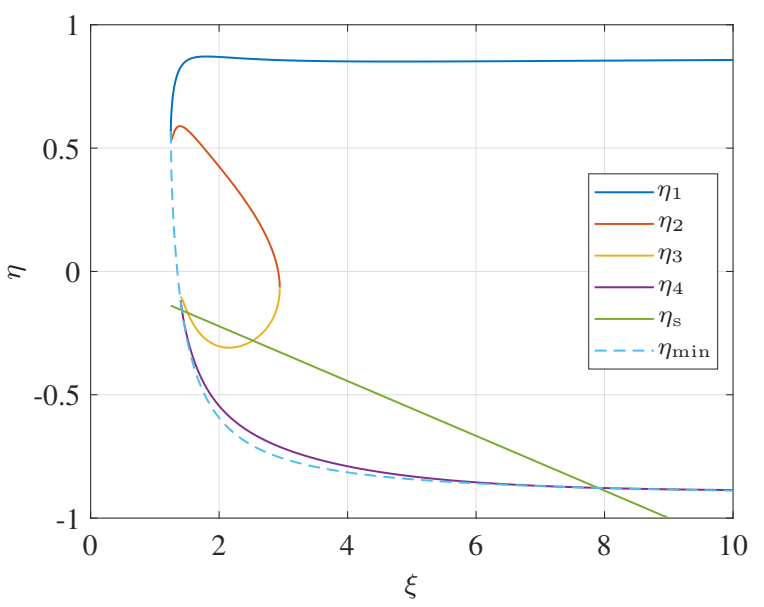

Fig. 4. Real roots $\eta_{1}, \eta_{2}, \eta_{3}, \eta_{4}$ of the polynomial, $\eta_{\mathrm{s}}$ of the singular point, and minimum value $\eta_{\min }$.

(29) become singular points. In this particular degenerate case, the extrema become cusps with values $\eta_{\min }$ or $\eta_{\max }$, since the algebraic curve degenerates into a line. The solutions are thus boundary values of the Doppler frequency, which are the minimum and maximum values of the Doppler pdf.

As in [33], we investigate closed-form solutions for the Doppler pdf and its corresponding characteristic function for the three different channel components mentioned by Bello in [18]: the LOS component, the SR component, and the diffuse scattering components.

\section{A. $\operatorname{LOS}$ Delay $(\xi=1)$}

The results for the LOS case concur with those obtained for the V2V channel in [33]. The Doppler pdf is naturally given by a Dirac distribution with the support at $f_{\text {los }}$ corresponding to the Doppler frequency caused by the LOS. As a result, the Doppler spread is zero and the characteristic function $\Phi(t ; u \mid \xi)$ is simply a complex exponential with the frequency given by $f_{\text {los. }}$. The stochastic channel functions are calculated as

$$
\begin{aligned}
& \Phi(t ; u \mid \xi)=\exp \left(\mathrm{j} 2 \pi u f_{\text {los }}\right), \\
& p\left(t ; f_{\mathrm{d}} \mid \xi\right)=\delta\left(f_{\mathrm{d}}-f_{\text {los }}\right), \\
& \mu(t ; \xi) \quad=\frac{v_{\mathrm{t} z}-v_{\mathrm{r} z}}{c} f_{\mathrm{c}}=f_{\text {los }}, \quad \sigma(t ; \xi)=0 .
\end{aligned}
$$

\section{Poles of the Doppler PDF close to LOS $(\xi \rightarrow 1)$}

In [33], we have shown that the poles of the pdf in the vicinity of LOS, i.e., as $\xi \rightarrow 1$, are located at the following frequencies:

$$
\begin{aligned}
& \lim _{\xi \rightarrow 1} f_{1,2}(t)=\frac{v_{\mathrm{t} z}-v_{\mathrm{r} z}}{c} f_{\mathrm{c}} \\
& \lim _{\xi \rightarrow 1} f_{3,4}(t)=\frac{ \pm\left\|\mathbf{v}_{\mathrm{t} \|}\right\|-v_{\mathrm{r} z}}{c} f_{\mathrm{c}}, \\
& \lim _{\xi \rightarrow 1} f_{5,6}(t)=\frac{ \pm\left\|\mathbf{v}_{\mathrm{r} \|}\right\|+v_{\mathrm{t} z}}{c} f_{\mathrm{c}} .
\end{aligned}
$$

In this case, the intersection of the ellipsoid with the ground plane has to be close to the major axis of the ellipsoid, i.e., 
when $\xi \rightarrow 1$. These scenarios are mainly relevant for taxiing, take-off, and landing. In these cases, the first double pole is found for $\eta \rightarrow \frac{v_{\mathrm{t} x}-v_{\mathrm{r} x}}{v_{\mathrm{t} x}+v_{\mathrm{r} x}}$ and the other four poles are obtained for $\eta \rightarrow \pm 1$. The contribution to the Doppler frequency, however, is only made by the velocity vector components $\mathbf{v}_{t \|}$ and $\mathbf{v}_{r \|}$ that are parallel to the ground plane.

The above equations are generally valid for 3D M2M channels when the ellipsoid is intersected with a scattering plane that includes the $z$-axis, i.e., TX and RX have to lie in the scattering plane in $\mathrm{A} 2 \mathrm{~A}$ and $\mathrm{V} 2 \mathrm{~V}$ scenarios.

\section{B. Specular reflection}

The specular reflection is in many respects similar to LOS case. In particular, the spectrum likewise consists of a Dirac distribution at the specular frequency $f_{\text {sr }}$ with a Doppler spread of zero. Also, in the vicinity of the specular reflection the spectrum "widens", which reflects close-to-reflection scattering; it then becomes wider as delay $\xi$ increases.

For the specular reflection we need to distinguish Casel and Case 2. Furthermore, if the aircraft are placed on the ground, i.e., $m=0$ and $d=0$, the specular reflection becomes the LOS consistent with the results of the V2V channel. As can be seen in Fig. 1, the specular reflection occurs, when the delay ellipsoid first touches the ground. From the geometry, the delay $\xi$ for the specular reflection can be obtained for both Case I and Case II. The calculated value for $\xi$ is then inserted into (7) to obtain the specular Doppler frequency $f_{\text {sr }}$. The delay and stochastic channel functions are calculated as follows

Case $1: \xi=\frac{a_{\mathrm{t}}+a_{\mathrm{r}}}{2 l} \quad$ or Case $2: \xi=\sqrt{\frac{d^{2}+l^{2}}{l^{2}+m^{2} l^{2}}}$
$\Phi(t ; u \mid \xi)=\exp \left(\mathrm{j} 2 \pi u f_{\mathrm{sr}}\right)$,
$p\left(t ; f_{\mathrm{d}} \mid \xi\right)=\delta\left(f_{\mathrm{d}}-f_{\mathrm{sr}}\right)$,
$\mu(t ; \xi)=f_{\mathrm{sr}}, \quad \sigma(t ; \xi)=0$
Case $1: f_{\mathrm{sr}}(t)= \pm \frac{v_{\mathrm{t} z}+v_{\mathrm{r} z}}{c} f_{\mathrm{c}}$
Case $2: f_{\mathrm{sr}}(t)=\frac{f_{\mathrm{c}}}{c} \sqrt{\frac{l^{2}+d^{2}}{1+m^{2}} \times}$
$\frac{l\left(v_{\mathrm{t} z}-v_{\mathrm{r} z}+m\left(v_{\mathrm{t} y}-v_{\mathrm{r} y}\right)\right)+d\left(v_{\mathrm{t} y}+v_{\mathrm{r} y}-m\left(v_{\mathrm{t} z}+v_{\mathrm{r} z}\right)\right)}{l^{2}+d^{2}}$

The sign convention for calculating $f_{\mathrm{sr}}(t)$ in Case 1 is the same as in (9). The positive sign is used, when the TX is above the RX and the negative sign, when the RX is above the TX.

\section{Distant delay $(\xi \rightarrow \infty)$}

For $\xi \rightarrow \infty$, the delay ellipsoid becomes a sphere and thus the intersection between delay ellipsoid and ground plane results in a circle. The circular symmetry then causes the Doppler pdf to become the well-known Jakes spectrum. For the distant delay case, we obtain the following results, which again concur with the V2V case.

$$
\begin{aligned}
& \lim _{\xi \rightarrow \infty} \Phi(t ; u \mid \xi)=J_{0}\left(2 \pi u \frac{\left\|\mathbf{v}_{\mathrm{t} \|}+\mathbf{v}_{\mathrm{r} \|}\right\|}{c} f_{\mathrm{c}}\right) \\
& \lim _{\xi \rightarrow \infty} p\left(t ; f_{\mathrm{d}} \mid \xi\right)=\frac{1}{\pi\left|f_{7,8}\right| \sqrt{1-\left(\frac{f_{\mathrm{d}}}{f_{7,8}}\right)^{2}}}, \\
& \lim _{\xi \rightarrow \infty} \mu(t ; \xi)=0, \quad \lim _{\xi \rightarrow \infty} \sigma(t ; \xi)=\frac{\left\|\mathbf{v}_{\mathrm{t} \|}+\mathbf{v}_{\mathrm{r} \|}\right\|}{\sqrt{2} c} f_{\mathrm{c}}, \\
& \lim _{\xi \rightarrow \infty} f_{7,8}(t)= \pm \frac{\left\|\mathbf{v}_{\mathrm{t} \|}+\mathbf{v}_{\mathrm{r} \|}\right\|}{c} f_{\mathrm{c}},
\end{aligned}
$$

The results depend, however, on the intersection of the ground plane with the ellipsoid. For airborne aircraft and scattering from the ground the equation reduces to the velocity vectors components $\mathbf{v}_{\mathrm{t} \|}$ and $\mathbf{v}_{\mathrm{r} \|}$ that are parallel to the ground plane. For Case 1 only the $v_{\mathrm{t} x}, v_{\mathrm{t} y}, v_{\mathrm{r} x} v_{\mathrm{r} y}$ components have an effect on the limiting frequencies of the Doppler spectrum. For equal altitudes in Case 2 only the $v_{\mathrm{t} x}, v_{\mathrm{t} z}$, $v_{\mathrm{r} x} v_{\mathrm{r} z}$ components are used in the calculation, which is in agreement with the results obtained for the V2V channel.

\section{REsults}

To compute synthetic A2A channels based on the developed theory, we numerically evaluate the presented equations. In order to generate the A2A channels, we use the intended future communication frequency for L-band Digital Aeronautical Communications System (LDACS) at $f_{\mathrm{c}}=1 \mathrm{GHz}$ [43]. Thus, the carrier frequency is in the aeronautical radio navigation service (ARNS) band that is currently used in civil aviation. The presented model does not take the effects of polarization into account. Usually, vertical polarized antennas are used in the aeronautical field . Our own measurements in [27] and [28] were likewise performed using vertically polarized antennas. We selected three typical aeronautical scenarios plus one artificial, which allows us to demonstrate a varying number of poles in the spectrum, which can be explained with the theory of algebraic curves. These scenarios are shown in Fig. 5 and the details of each scenario are summarized in the list below:

- Scenario 1 (Fig. 5a): Two aircraft flying at equal altitudes $a_{\mathrm{t}}=a_{\mathrm{r}}=1000 \mathrm{ft}$ at a distance of $2 l=2 \mathrm{~nm}$ with velocity vectors $\mathbf{v}_{\mathrm{t}}=[0,0,900]^{\mathrm{T}} \mathrm{km} / \mathrm{h}$ and $\mathbf{v}_{\mathrm{r}}=$ $[0,0,900]^{\mathrm{T}} \mathrm{km} / \mathrm{h}$. The slope is $m=0$ and the ratio of $y$-intercept to focus distance is $d / l=0.1646$.

- Scenario 2 (Fig. 5b): Two aircraft performing a vertical pass-by. TX is at $a_{\mathrm{t}}=1000 \mathrm{ft}$ and $\mathrm{RX}$ at $a_{\mathrm{r}}=2000 \mathrm{ft}$. Planes are flying on orthogonal trajectories with velocity vectors $\mathbf{v}_{\mathrm{t}}=[900,0,0]^{\mathrm{T}} \mathrm{km} / \mathrm{h}$ and $\mathbf{v}_{\mathrm{r}}=[0,-900,0]^{\mathrm{T}} \mathrm{km} / \mathrm{h}$. The ratio of $z$-intercept to focus distance is $-\frac{a_{\mathrm{t}}+a_{\mathrm{r}}}{2 l}=-3$. This is the only scenario, which covers Case I.

- Scenario 3 (Fig. 5c): Two aircraft landing on two parallel runways. The runway separation is $915 \mathrm{~m}$. The aircraft are separated by $2 l=2 \mathrm{~nm}$. The aircraft altitudes are $a_{\mathrm{t}}=1617 \mathrm{ft}$ and $a_{\mathrm{r}}=1000 \mathrm{ft}$ The glide slope is $3^{\circ}$ with velocity vectors $\mathbf{v}_{\mathrm{t}}=[74,0,291]^{\mathrm{T}} \mathrm{km} / \mathrm{h}$ and $\mathbf{v}_{\mathrm{r}}=$ 


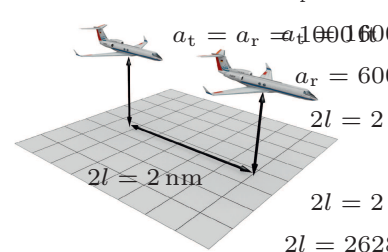

(a) Scenario 1 .

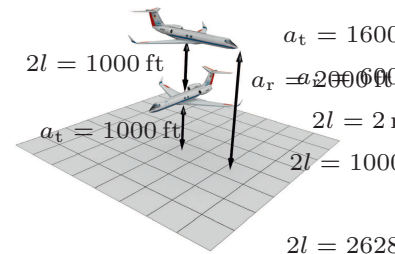

(b) Scenario 2 .

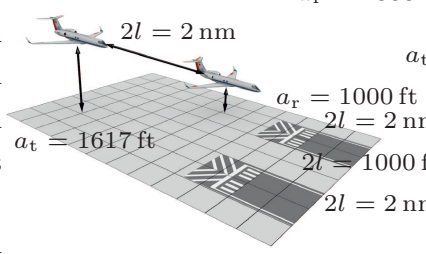

(c) Scenario 3.

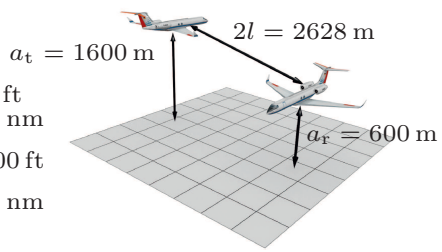

(d) Scenario 4 .

Fig. 5. Graphical sketches of the four simulation scenarios. The parameters of the scenarios are found in the scenario description below.

$[74,0,291]^{\mathrm{T}} \mathrm{km} / \mathrm{h}$. The slope is $m=-0.0508$ and the ratio of $y$-intercept to focus distance is $d / l=0.2156$.

- Scenario 4 (Fig. 5d): Two aircraft are flying at different altitudes and with different velocity vectors. TX is at $a_{\mathrm{t}}=1600 \mathrm{~m}$ and RX at $a_{\mathrm{r}}=600 \mathrm{~m}$ at a distance of $2 l=$ $2628 \mathrm{~m}$ with velocity vectors $\mathbf{v}_{\mathrm{t}}=[200,-19,46]^{\mathrm{T}} \mathrm{km} / \mathrm{h}$ and $\mathbf{v}_{\mathrm{r}}=[-250,-38,92]^{\mathrm{T}} \mathrm{km} / \mathrm{h}$. The slope is $m=$ -0.4115 and the ratio of $y$-intercept to focus distance is $d / l=0.9053$. This scenario is introduced to show a varying number of poles and a crunode in the Doppler frequency.

In this paper, $\mathrm{nm}$ is referring to the unit nautical mile. Although we provided the scenarios with specific values of distance and altitude, the results are the same for all scenarios with the same slope $m$ and the same ratio of axis intercept to focus distance $d / l$. Thus, the results are general and apply also for larger aircraft distances, if they fly at higher altitudes. The presented scenarios are all snapshots in time. If the reader is interested in how the model behaves when time elapses, we recommend to have a look at [44]. There, the aircraft fly on different altitudes past each other. Subsequently, we consider the listed scenarios in more details.

Scenario 1: In this case, the delay-dependent Doppler pdf shows a round shape for fixed $t=t^{*}$ and small $\xi$. As explained above, the delay-dependent Doppler pdfs for fixed $\xi=\xi^{*}$ in Fig. 8 grows wider for larger delays. The specular reflection frequency is calculated as $f_{\mathrm{sr}}\left(t^{*}\right)=0 \mathrm{~Hz}$. Furthermore the typical W-shape of the Doppler spectrum for A2A channels becomes apparent. The widening of the delay-dependent Doppler pdf leads to a narrowing characteristic function in Fig. 9, which means the channel is more time-variant for larger delays. The symmetry of the delay-dependent Doppler pdf is due to the fact that both aircraft fly along the major axis of the ellipsoid with the same speed. The delay-dependent limits of the Doppler pdf are calculated by solving the $6^{\text {th }}$ order polynomial in (29). There are only two extreme values, since in Scenario 1 the degenerate case occurs as described in Section III. This means the extrema are boundary values of the algebraic curve and thus cusps. The algebraic curve degenerates into a straight line as can be seen in Fig. 7. The polynomial, however, still determines the location of the boundary values correctly, even though the partial derivative $\partial f_{\mathrm{d}} / \partial \eta \neq 0$. Without the theory of algebraic curves, it cannot be explained, why the root of the polynomial correctly determines the boundary values. The locations of the boundary frequencies are shown in Fig. 6 as dashed line. For large $\xi$

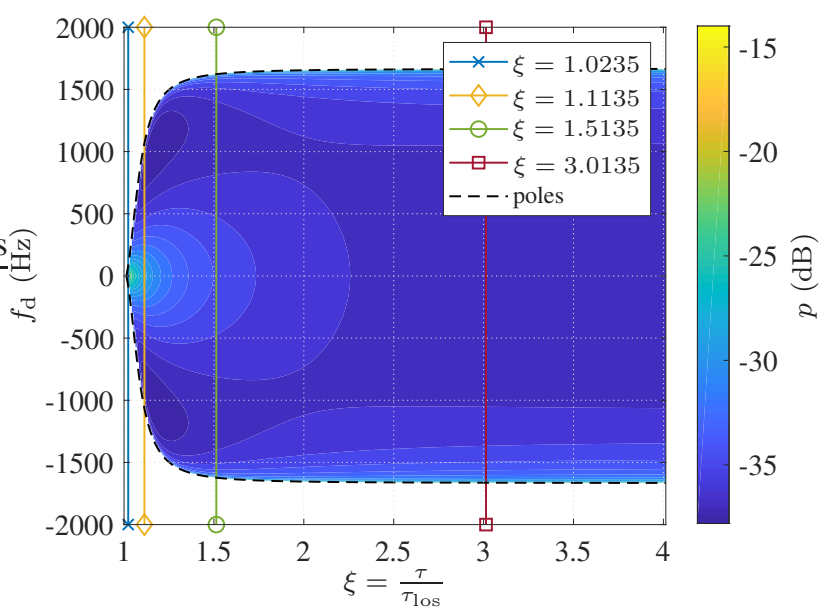

Fig. 6. Scenario 1: General delay-dependent Doppler pdf $p\left(t^{*} ; f_{\mathrm{d}} \mid \xi\right)$ for $\mathbf{v}_{\mathrm{t}}=[0,0,900]^{\mathrm{T}} \mathrm{km} / \mathrm{h}$ and $\mathbf{v}_{\mathrm{r}}=[0,0,900]^{\mathrm{T}} \mathrm{km} / \mathrm{h}$ at a distance of $2 l=$ $2 \mathrm{~nm}$.

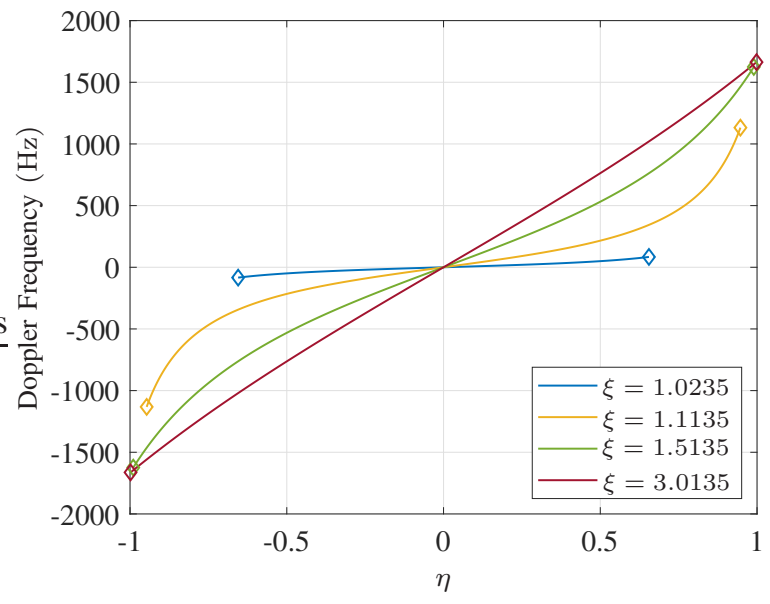

Fig. 7. Scenario 1: Degenerate sextic curve $\mathcal{C}\left(f_{\mathrm{d}}, \eta\right)$, where the cusps are the boundary values of the Doppler frequency marked by a diamond.

the spectral boundaries tend towards $f_{7,8}\left(t^{*}\right)= \pm 1667 \mathrm{~Hz}$ in Fig. 6 and the characteristic function becomes a Bessel function in Fig. 9.

Scenario 2: The delay-dependent Doppler pdf in Fig. 10 shows an even rounder shape due to the circular intersection of the vertical ellipsoid with the ground. This is explained by the circular symmetry, since the vertical ellipsoid cuts the ground plane as a circle. The specular reflection frequency 


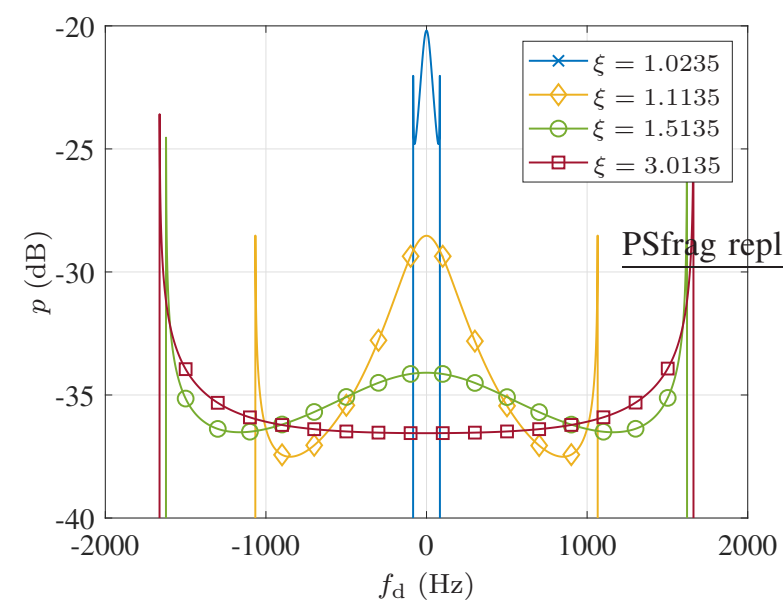

Fig. 8. Scenario 1: Specific delay-dependent Doppler pdfs $p\left(t^{*} ; f_{\mathrm{d}} \mid \xi^{*}\right)$ for $\mathbf{v}_{\mathrm{t}}=[0,0,250]^{\mathrm{T}} \mathrm{km} / \mathrm{h}$ and $\mathbf{v}_{\mathrm{r}}=[0,0,250]^{\mathrm{T}} \mathrm{km} / \mathrm{h}$ at a distance of $2 l=$ $2 \mathrm{~nm}$.

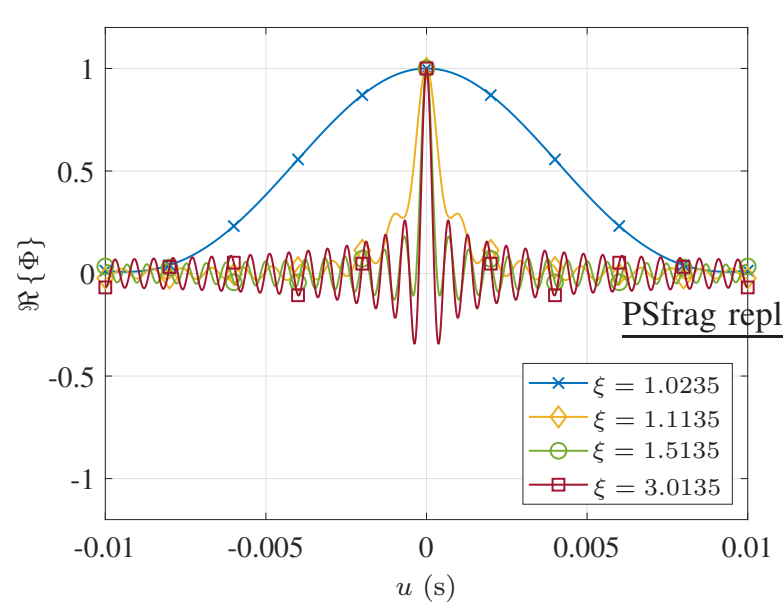

Fig. 9. Scenario 1: Real part of specific delay-dependent characteristic functions $\Re\left\{\Phi\left(t^{*} ; u \mid \xi^{*}\right)\right\}$ for $\mathbf{v}_{\mathrm{t}}=[0,0,900]^{\mathrm{T}} \mathrm{km} / \mathrm{h}$ and $\mathbf{v}_{\mathrm{r}}=$ $[0,0,900]^{\mathrm{T}} \mathrm{km} / \mathrm{h}$ at a distance of $2 l=2 \mathrm{~nm}$.

is calculated as $f_{\mathrm{sr}}\left(t^{*}\right)=0 \mathrm{~Hz}$, since there is no vertical velocity component. The delay-dependent Doppler pdfs for fixed $\xi=\xi^{*}$ in Fig. 11 grows wider for increasing delays $\xi$. The delay-dependent limits of the Doppler spectrum are calculated by (29) and shown as dashed lines in Fig. 10. All the Doppler spectra look like Jakes spectra, which is clearly due to the circular symmetry of the angular distribution in (11). The widening of the Doppler pdf leads to a narrowing Bessel function in Fig. 12, which means higher time-variance. For $\xi=5$ the spectral limits tend already towards $f_{7,8}\left(t^{*}\right)= \pm 1179 \mathrm{~Hz}$ as can be seen in Fig. 11.

Scenario 3: The delay-dependent Doppler pdf in Fig. 13 shows a round asymmetric shape due to the different altitudes of the aircraft. As in the previous scenarios, the Doppler pdfs for fixed $\xi=\xi^{*}$ in Fig. 14 grows wider for increasing delays $\xi$. The specular reflection frequency is calculated as $f_{\mathrm{sr}}\left(t^{*}\right)=6 \mathrm{~Hz}$. The first two Doppler pdfs, i.e., for $\xi=1.0317$ and $\xi=1.1217$, show an asymmetric W-shape for this

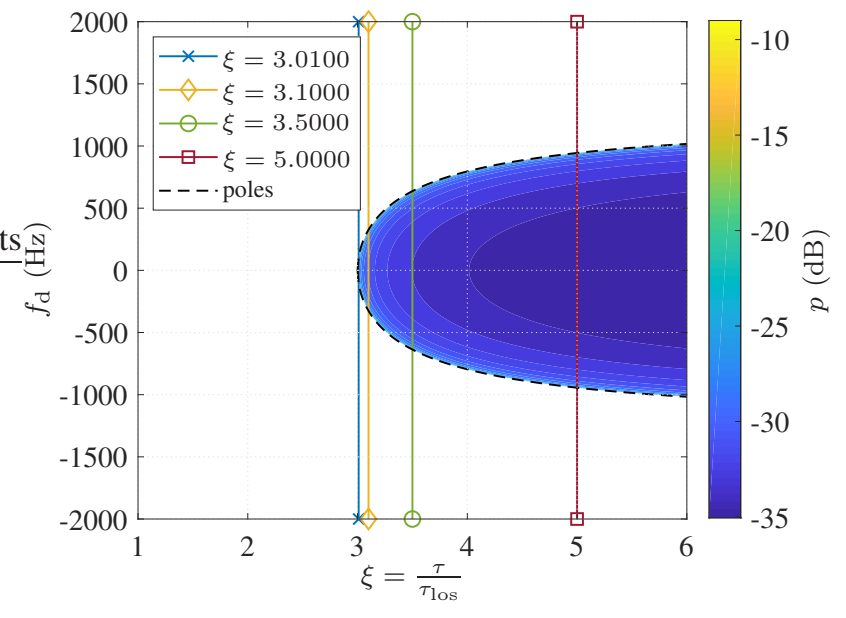

Fig. 10. Scenario 2: General delay-dependent Doppler pdf $p\left(t^{*} ; f_{\mathrm{d}} \mid \xi\right)$ for $\mathbf{v}_{\mathrm{t}}=[900,0,0]^{\mathrm{T}} \mathrm{km} / \mathrm{h}$ and $\mathbf{v}_{\mathrm{r}}=[0,-900,0]^{\mathrm{T}} \mathrm{km} / \mathrm{h}$ at a distance of $2 l=1000 \mathrm{ft}$.

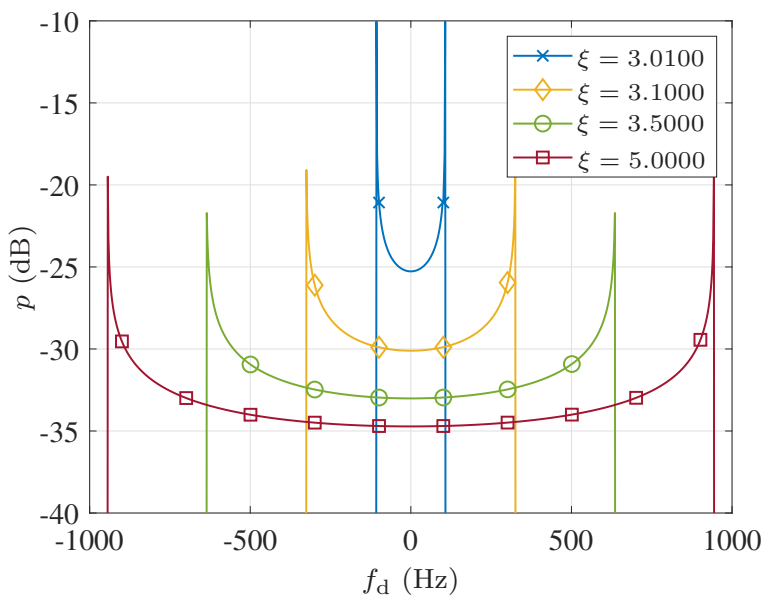

Fig. 11. Scenario 2: Specific delay-dependent Doppler pdfs $p\left(t^{*} ; f_{\mathrm{d}} \mid \xi^{*}\right)$ for $\mathbf{v}_{\mathrm{t}}=[900,0,0]^{\mathrm{T}} \mathrm{km} / \mathrm{h}$ and $\mathbf{v}_{\mathrm{r}}=[0,-900,0]^{\mathrm{T}} \mathrm{km} / \mathrm{h}$ at a distance of $2 l=1000 \mathrm{ft}$.

scenario. The last Doppler pdf for $\xi=3.0217$ exhibits the typical Jakes shape. The location of the poles are displayed as dashed lines in Fig. 13. The widening of the Doppler pdf leads to a narrowing characteristic function in Fig. 15 and finally to a Bessel function for large delays. For large $\xi$ the spectral limits tend towards $f_{7,8}\left(t^{*}\right)= \pm 555 \mathrm{~Hz}$ in Fig. 14 .

Scenario 4: This particular scenario was specially selected to explain the application of the theory of algebraic curves with the three different types of singular points. The delaydependent Doppler pdf shown in Fig. 16 shows a round shape close to the specular reflection. Due to the different altitudes and velocity vectors of TX and RX the shape is asymmetric. The specular reflection frequency is calculated as $f_{\mathrm{sr}}\left(t^{*}\right)=-34 \mathrm{~Hz}$. The Doppler pdf for fixed $\xi=\xi^{*}$ in Fig. 17 grows wider and consists of one $(\xi=1.2474)$, two $(\xi=1.3474, \xi=3.2474)$ or three parts $(\xi=1.7474)$. The different parts of the spectrum all exhibit the classical Jakes shape. This knowledge could be used, when estimating the spectrum. The widening of the Doppler pdf leads to a 


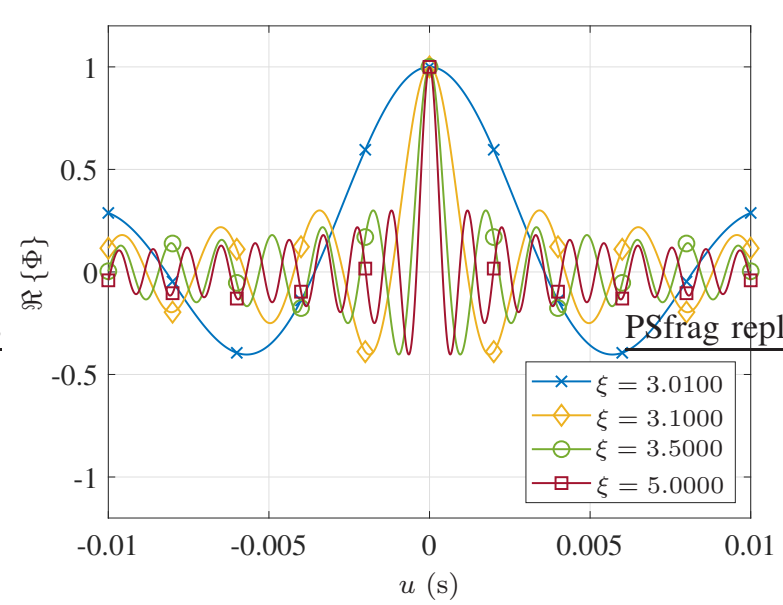

Fig. 12. Scenario 2: Real part of specific delay-dependent characteristic functions $\Re\left\{\Phi\left(t^{*} ; u \mid \xi^{*}\right)\right\}$ for $\mathbf{v}_{\mathrm{t}}=[900,0,0]^{\mathrm{T}} \mathrm{km} / \mathrm{h}$ and $\mathbf{v}_{\mathrm{r}}=$ $[0,-900,0]^{\mathrm{T}} \mathrm{km} / \mathrm{h}$ at a distance of $2 l=1000 \mathrm{ft}$.

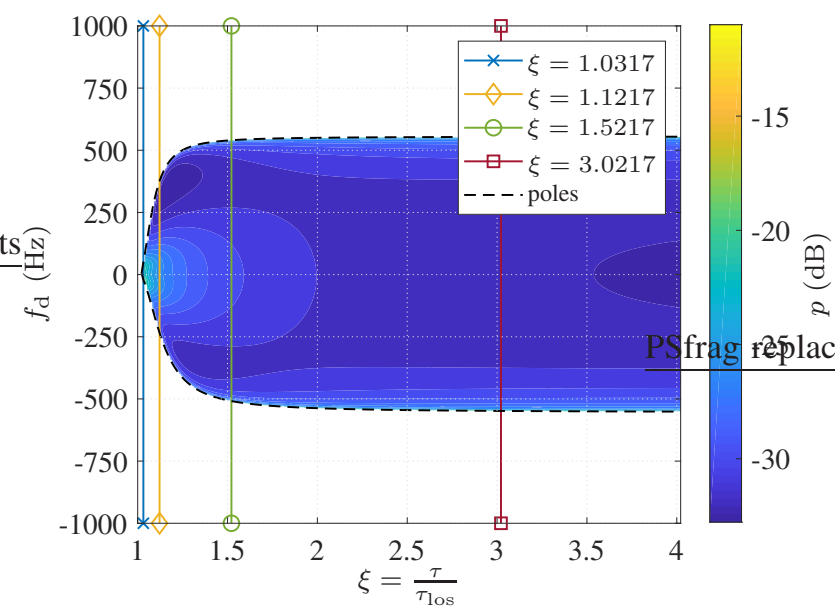

Fig. 13. Scenario 3: General delay-dependent Doppler pdf $p\left(t^{*} ; f_{\mathrm{d}} \mid \xi\right)$ for $\mathbf{v}_{\mathrm{t}}=[74,0,291]^{\mathrm{T}} \mathrm{km} / \mathrm{h}$ and $\mathbf{v}_{\mathrm{r}}=[74,0,291]^{\mathrm{T}} \mathrm{km} / \mathrm{h}$. The aircraft are separated by $2 l=2 \mathrm{~nm}$. The glide slope is $3^{\circ}$.

narrowing characteristic function in Fig. 18. For large $\xi$ the spectral limits tend towards $f_{7,8}\left(t^{*}\right)= \pm 139 \mathrm{~Hz}$. The delaydependent width of the Doppler spectrum is calculated by solving the $6^{\text {th }} t h$ order polynomial in (29). The poles in the delay-dependent Doppler spectrum are shown as dashed lines in Fig. 16 and possess a whale-like shape. In this special scenario, the number of real roots increases from two to four between the delays $\xi=1.422$ and $\xi=2.943$. For larger delays, the number decreases again to two. The number of poles depends on the number of extrema of the algebraic Doppler curve, which is shown in Fig. 3 in Section III. Between the delays $\xi=1.422$ and $\xi=2.943$, the curve possesses four extrema and crosses itself once. For delays $2.943<\xi<7.987$, the curve only has two extrema, but still crosses itself once.

\section{CONCLUSION}

In this paper, we have derived a three-dimensional, singlebounce scattering model for 3D aircraft-to-aircraft channels.

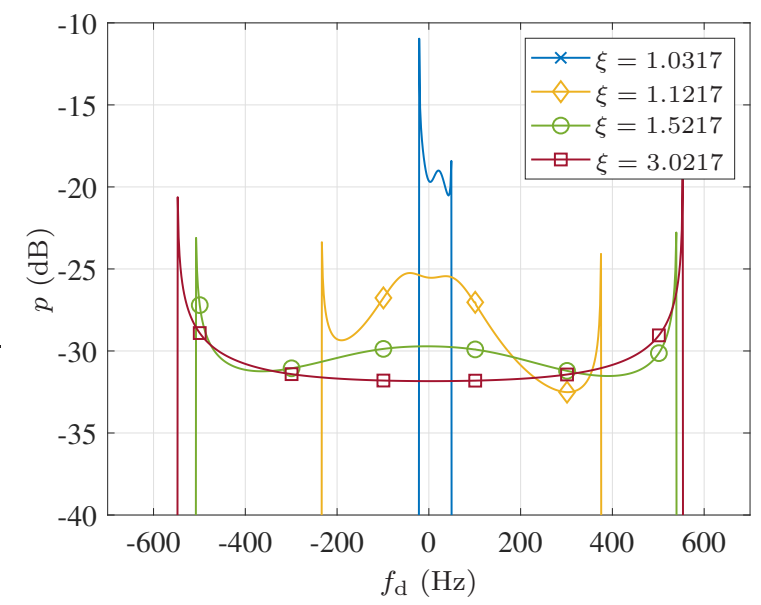

Fig. 14. Scenario 3: Specific delay-dependent Doppler pdfs $p\left(t^{*} ; f_{\mathrm{d}} \mid \xi^{*}\right)$ for $\mathbf{v}_{\mathrm{t}}=[74,0,291]^{\mathrm{T}} \mathrm{km} / \mathrm{h}$ and $\mathbf{v}_{\mathrm{r}}=[74,0,291]^{\mathrm{T}} \mathrm{km} / \mathrm{h}$. The aircraft are separated by $2 l=2 \mathrm{~nm}$. The glide slope is $3^{\circ}$.

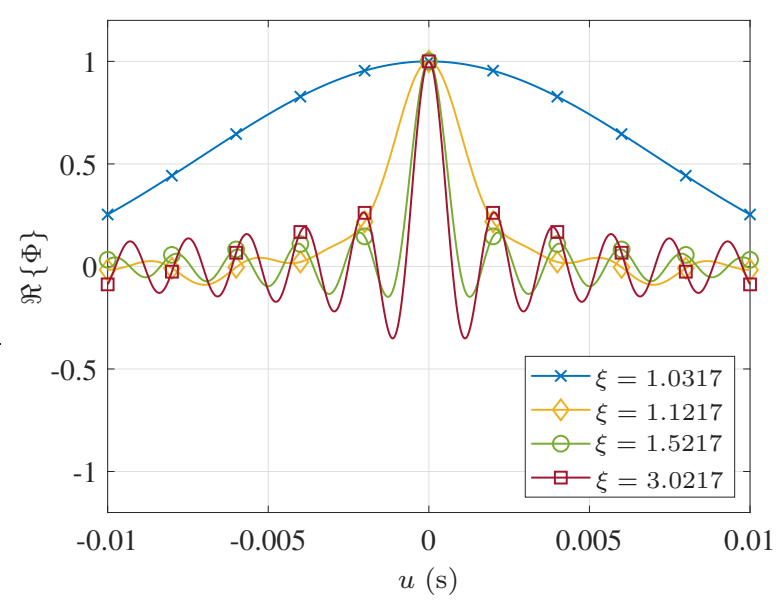

Fig. 15. Scenario 3: Real part of specific delay-dependent characteristic functions $\Re\left\{\Phi\left(t^{*} ; u \mid \xi^{*}\right)\right\}$ for $\mathbf{v}_{\mathrm{t}}=[74,0,291]^{\mathrm{T}} \mathrm{km} / \mathrm{h}$ and $\mathbf{v}_{\mathrm{r}}=$ $[74,0,291]^{\mathrm{T}} \mathrm{km} / \mathrm{h}$. The aircraft are separated by $2 l=2 \mathrm{~nm}$. The glide slope is $3^{\circ}$.

Similar to vehicle-to-vehicle channels, the derived model is likewise time-variant and provides a description of nonstationary aircraft-to-aircraft channels. Time-variant limiting frequencies of the delay-dependent Doppler probability function were derived by an algebraic analysis of the Doppler frequency in prolate spheroidal coordinates. These frequencies were shown to be the solutions of a sixth degree polynomial, which concurs with the vehicle-to-vehicle channel. The algebraic curve analysis reveals the presence of singular points, which might become extreme points, i.e., boundary values of the curve. Thus, the time-variant boundaries of the Doppler pdf can be seamlessly calculated for arbitrary delays and aircraft configurations.

The obtained results readily generalize those obtained for $2 \mathrm{D}$ vehicle-to-vehicle scenarios. They can be used to account for arbitrary combinations of both rotary as well as fixed-wing aircraft and ground-based vehicles in similar environments. 


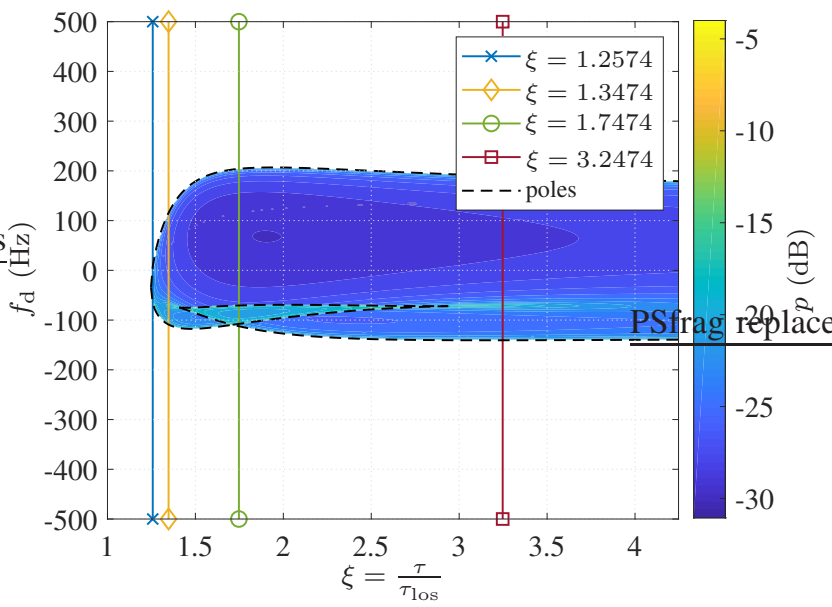

Fig. 16. Scenario 4: General delay-dependent Doppler pdf $p\left(t^{*} ; f_{\mathrm{d}} \mid \xi\right)$ for $\mathbf{v}_{\mathrm{t}}=[200,-19,46]^{\mathrm{T}} \mathrm{km} / \mathrm{h}$ and $\mathbf{v}_{\mathrm{r}}=[-250,-38,92]^{\mathrm{T}} \mathrm{km} / \mathrm{h}$ at a distance of $2 l=2628 \mathrm{~m}$.

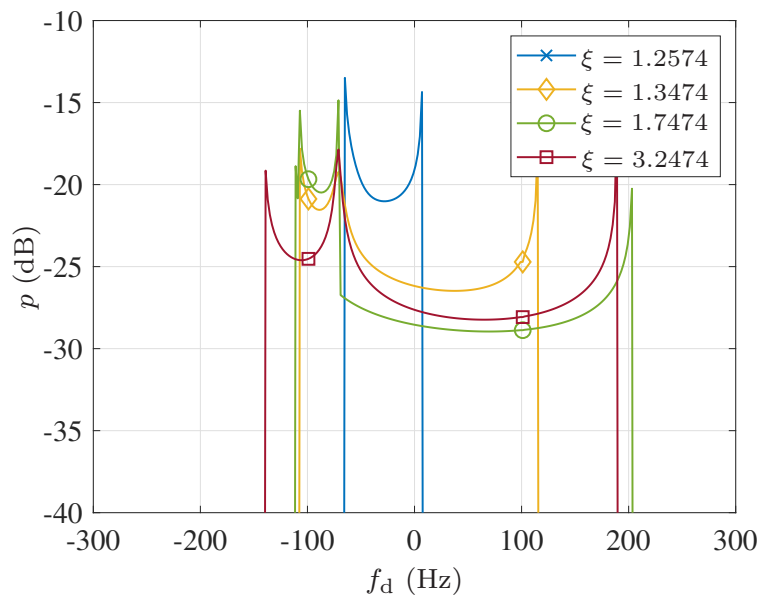

Fig. 17. Scenario 4: Specific delay-dependent Doppler pdfs $p\left(t^{*} ; f_{\mathrm{d}} \mid \xi *\right)$ $\mathbf{v}_{\mathrm{t}}=[200,-19,46]^{\mathrm{T}} \mathrm{km} / \mathrm{h}$ and $\mathbf{v}_{\mathrm{r}}=[-250,-38,92]^{\mathrm{T}} \mathrm{km} / \mathrm{h}$ at a distance of $2 l=2628 \mathrm{~m}$.

\section{REFERENCES}

[1] J. Qiao, X. S. Shen, J. W. Mark, Q. Shen, Y. He, and L. Lei, "Enabling device-to-device communications in millimeter-wave $5 \mathrm{G}$ cellular networks," IEEE Commun. Mag., vol. 53, no. 1, pp. 209-215, Jan. 2015.

[2] M. Walter, N. Franzen, and M. Schnell, "New concepts for a decentralized, self-organizing air-to-air radio link," in Proc. IEEE/AIAA 29th Digital Avionics Syst. Conf.(DASC), Salt Lake City, UT, USA, Oct. 2010, pp. 3.D.1-1-3.D.1-12.

[3] N. Goddemeier and C. Wietfeld, "Investigation of air-to-air channel characteristics and a UAV specific extension to the Rice model," in Proc. IEEE Global Telecommun. Conf. (GLOBECOM) Workshops, Dec. 2015, pp. $1-5$.

[4] R. H. Clarke, "A statistical theory of mobile-radio reception," Bell Syst. Tech. J., vol. 47, no. 6, pp. 957-1000, Jul./Aug. 1968.

[5] P. A. Bello, "Characterization of randomly time-variant linear channels," IEEE Trans. Commun., vol. 11, no. 4, pp. 360-393, Dec. 1963.

[6] W. C. Jakes, Microwave Mobile Communications, ser. IEEE Press Classic Reissue. New York, USA: Wiley, 1994.

[7] A. S. Akki and F. Haber, "A statistical model of mobile-to-mobile land communication channel," IEEE Trans. Veh. Technol., vol. 35, no. 1, pp. 2-7, Feb. 1986.

[8] F. Vatalaro and A. Forcella, "Doppler spectrum in mobile-to-mobile communications in the presence of three-dimensional multipath scat-

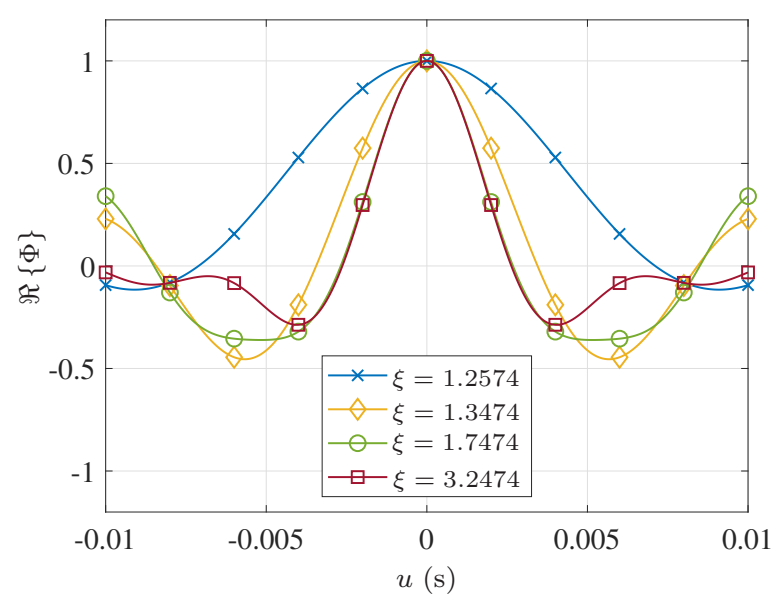

Fig. 18. Scenario 4: Real part of specific delay-dependent characteristic functions $\Re\left\{\Phi\left(t^{*} ; u \mid \xi^{*}\right)\right\}$ for $\mathbf{v}_{\mathrm{t}}=[200,-19,46]^{\mathrm{T}} \mathrm{km} / \mathrm{h}$ and $\mathbf{v}_{\mathrm{r}}=$ $[-250,-38,92]^{\mathrm{T}} \mathrm{km} / \mathrm{h}$ at a distance of $2 l=2628 \mathrm{~m}$.

tering," IEEE Trans. Veh. Technol., vol. 46, no. 1, pp. 213-219, Feb. 1997.

[9] C. S. Patel, G. L. Stüber, and T. G. Pratt, "Simulation of Rayleigh-faded mobile-to-mobile communication channels," IEEE Trans. Commun., vol. 53, no. 11, pp. 1876-1884, Nov. 2005.

[10] Z. Tang and A. S. Mohan, "A correlated indoor MIMO channel model," in Canadian Conf. Elect. Comput. Eng., Montreal, QC, Canada, May 2003, pp. $1889-1892$.

[11] A. G. Zajić and G. L. Stüber, "3-D MIMO mobile-to-mobile channel simulation," in 2007 16th IST Mobile and Wireless Commun. Summit, Budapest, Hungary, Jul. 2007, pp. 1-5.

[12] M. Pätzold, B. O. Hogstad, N. Youssef, and D. Kim, "A MIMO mobileto-mobile channel model: Part I - the reference model," in Proc. IEEE 16th Int. Symp. Personal, Indoor and Mobile Radio Commun., Berlin, Germany, Sep. 2005, pp. 573-578.

[13] A. Abdi and M. Kaveh, "A space-time correlation model for multielement antenna systems in mobile fading channels," IEEE J. Select. Areas Commun., vol. 20, no. 3, pp. 550-560, Apr. 2002.

[14] M. Pätzold, B. O. Hogstad, and N. Youssef, "Modeling, analysis, and simulation of MIMO mobile-to-mobile fading channels," IEEE Trans. Wireless Commun., vol. 7, no. 2, pp. 510-520, Feb. 2008

[15] A. G. Zajić and G. L. Stüber, "Three-dimensional modeling, simulation, and capacity analysis of space-time correlated mobile-to-mobile channels," IEEE Trans. Veh. Technol., vol. 57, no. 4, pp. 2042-2054, Jul. 2008.

[16] A. G. Zajić, G. L. Stüber, T. G. Pratt, and S. Nguyen, "Statistical modeling and experimental verification of wideband MIMO mobile-tomobile channels in highway environments," in Proc. IEEE 19th Int. Symp. Personal, Indoor and Mobile Radio Commun., Cannes, France, Sep. 2008, pp. 1-5.

[17] E. Haas, "Aeronautical channel modeling," IEEE Trans. Veh. Technol., vol. 51, no. 2, pp. 254-264, Mar. 2002.

[18] P. A. Bello, "Aeronautical channel characterization," IEEE Trans. Commun., vol. 21, no. 5, pp. 548-563, May 1973.

[19] A. Paier, T. Zemen, L. Bernadó, G. Matz, J. Karedal, N. Czink, C. Dumard, F. Tufvesson, A. F. Molisch, and C. F. Mecklenbräuker, "Non-WSSUS vehicular channel characterization in highway and urban scenarios at $5.2 \mathrm{GHz}$ using the local scattering function," in Int. ITG Workshop on Smart Antennas (WSA), Darmstadt, Germany, Feb. 2008, pp. 9-15.

[20] L. Bernadó, "Non-stationarity in vehicular wireless channels," Ph.D. dissertation, Technische Universität Wien, Vienna, Austria, Apr. 2012.

[21] G. Matz, "On non-WSSUS wireless fading channels," IEEE Trans. Wireless Commun., vol. 4, no. 5, pp. 2465-2478, Sep. 2005.

[22] J. Karedal, F. Tufvesson, N. Czink, A. Paier, C. Dumard, T. Zemen, C. F. Mecklenbräuker, and A. F. Molisch, "A geometry-based stochastic MIMO model for vehicle-to-vehicle communications," IEEE Trans. Wireless Commun., vol. 8, no. 7, pp. 3646-3657, Jul. 2009.

[23] M. Walter, D. Shutin, and U.-C. Fiebig, "Delay-dependent Doppler probability density functions for vehicle-to-vehicle scatter channels," 
IEEE Trans. Antennas Propagat., vol. 62, no. 4, pp. 2238-2249, Apr. 2014.

[24] — - "Joint delay Doppler probability density functions for air-to-air channels," Int. J. Antennas Propagat., vol. 2014, pp. 1-11, 2014.

[25] M. Walter, U.-C. Fiebig, and A. Zajić, "Experimental verification of the non-stationary statistical model for V2V scatter channels," in Proc. IEEE 80th Veh. Technol. Conf., Vancouver, BC, Canada, Sep. 2014, pp. $1-5$.

[26] M. Walter, T. Zemen, and D. Shutin, "Empirical relationship between local scattering function and joint probability density function," in Proc. IEEE 26th Int. Symp. Personal, Indoor and Mobile Radio Commun., Hong Kong, China, Aug. 2015.

[27] M. Walter, "Scattering in non-stationary mobile-to-mobile communications channels," Ph.D. dissertation, Universität Ulm, 2016.

[28] M. Walter and M. Schnell, "The Doppler-delay characteristic of the aeronautical scatter channel," in Proc. IEEE 74th Veh. Technol. Conf. (VTC Fall), San Francisco, CA, USA, Sep. 2011, pp. 1-5.

[29] W. G. Newhall and J. H. Reed, "A geometric air-to-ground radio channel model," in Proc. IEEE Military Commun. Conf. (MILCOM), Anaheim, CA, USA, Oct. 2002, pp. 632-636.

[30] M. Ibrahim and H. Arslan, "Air-ground Doppler-delay spread spectrum for dense scattering environments," in Proc. IEEE Military Commun. Conf. (MILCOM), Tampa, FL, USA, Oct. 2015, pp. 1661-1666.

[31] S. M. Gulfam, S. J. Nawaz, A. Ahmed, M. N. Patwary, and Q. Ni, "A novel 3D analytical scattering model for air-to-ground fading channels," Applied Sciences, vol. 6, no. 207, 2016.

[32] M. Walter, D. Shutin, and U.-C. Fiebig, "Prolate spheroidal coordinates for modeling mobile-to-mobile channels," IEEE Antennas Wireless Propagat. Lett., vol. 14, pp. 155-158, 2015.

[33] M. Walter, D. Shutin, and A. Dammann, "Time-variant Doppler PDFs and characteristic functions for the vehicle-to-vehicle channel," IEEE Trans. Veh. Technol., vol. 66, no. 12, pp. 10748-10 763, Dec. 2017.

[34] _ , "Algebraic analysis of the poles in the Doppler spectrum for vehicle-to-vehicle channels," IEEE Wireless Commun. Lett., vol. 7, no. 4, pp. 662-665, Aug. 2018.

[35] C. Flammer, Spheroidal Wave Functions. Stanford, CA: Stanford University Press, 1957.

[36] I. N. Bronstein, K. A. Semendyayev, G. Musiol, and H. Mühlig, Handbook of Mathematics. Springer, 2007.

[37] A. Zajić, Mobile-to-Mobile Wireless Channels, 1st ed. Boston, MA, USA: Artech House, 2012.

[38] A. Papoulis and S. U. Pillai, Probability, Random Variables, and Stochastic Processes, ser. McGraw-Hill electrical and electronic engineering series. McGraw-Hill, 2002.

[39] M. Pätzold, Mobile Fading Channels. Chichester, UK: Wiley, 2002.

[40] H. Hilton, Plane algebraic curves. The Clarendon press, 1920.

[41] S. S. Abhyankar, Algebraic Geometry for Scientists and Engineers, ser. Mathematical Surveys and Monographs. American Mathematical Society, 1990.

[42] T. R. Hagedorn, "General formulas for solving solvable sextic equations," Journal of Algebra, vol. 233, no. 2, pp. 704 - 757, 2000.

[43] M. Schnell, U. Epple, D. Shutin, and N. Schneckenburger, "LDACS: future aeronautical communications for air-traffic management," IEEE Commun. Mag., vol. 52, no. 5, pp. 104-110, May 2014.

[44] M. Walter, D. Shutin, A. Dammann, and D. W. Matolak, "Modeling of highly non-stationary low altitude aircraft-to-aircraft channels," in Proc. IEEE Military Commun. Conf. (MILCOM), Los Angeles, CA, USA, Oct. 2018.

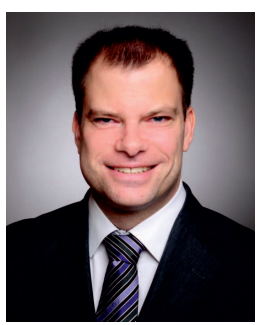

Michael Walter (M'10-SM'17) received his Dipl.Ing. and Dr.-Ing. degree in electrical engineering from Ulm University, Germany, in 2008 and 2015.

Since 2009 he has been a member of the scientific staff at the Institute of Communications and Navigation at the German Aerospace Center (DLR) in Oberpfaffenhofen, Germany, where he gained extensive experience in organizing and conducting numerous measurement campaigns for vehicular and aeronautical research. His research interests include wireless communications, modeling of mobile-tomobile channels, and channel coding.

Dr. Walter was the recipient of the IEEE/AIAA David Lubkowski Memorial for Advancement in Digital Avionics Best Paper Award of the 29th DASC in 2011.

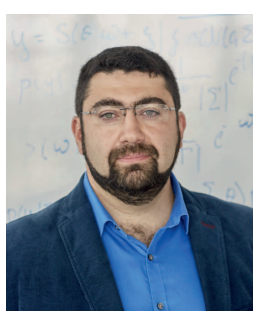

Dmitriy Shutin (M'06-SM'12) received his Master degree in Computer Science in 2000 from Dniepropetrovsk State University, Ukraine, and the $\mathrm{Ph} . \mathrm{D}$. degree in electrical engineering from Graz University of Technology, Graz, Austria, in 2006.

During 2001-2006 and 2006-2009, he was a Teaching Assistant and an Assistant Professor, respectively, with the Signal Processing and Speech Communication Laboratory, Graz University of Technology. From 2009 to 2011 he was a Research Associate with the Department of Electrical Engineering at Princeton University, Princeton, NJ, USA. Since 2011 he is with the Institute of Communications and Navigation, German Aerospace Center. His current research interests include modeling and estimation of the radio propagation channels, statistical signal processing, radar signal processing, and machine learning for signal processing.

Dr. Shutin was a recipient of the Best Student Paper Award at the 2005 IEEE International Conference on Information, Communications and Signal Processing (ICICS). In 2009 he was awarded the Erwin Schroedinger Research Fellowship.

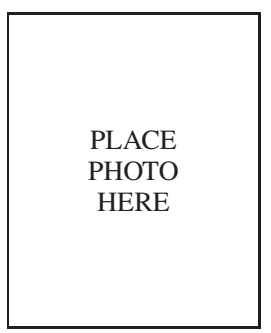

David W. Matolak (S'82, M'83, SM'00) was born in Johnstown, PA. He received the B.S. degree from The Pennsylvania State University, University Park, PA, the M.S. degree from The University of Massachusetts, Amherst, MA, and the Ph.D. degree from The University of Virginia, Charlottesville, VA, all in electrical engineering. He worked with the Rural Electrification Administration, Washington, D.C., on upgrading of specialized rural telecommunication systems, and with the UMass LAMMDA Laboratory on the full-wave analysis, design, fabrication, and testing of planar microwave transmission lines and antennas. He spent three years with AT\&T Bell Laboratories in the Microwave Radio Systems Development Department, where he worked on the analytical and empirical characterization of nonlinearities and their effect on QAM transmission. As a Ph.D. student in the University of Virginia's Communication Systems Laboratory, he focused on analysis of trellis coding and equalization for TDMA mobile radio systems. He spent two years with Lockheed Martin Tactical Communication Systems, as Lead System Engineer on the development of a wireless local loop synchronous code division multiple access (CDMA) communication system. Following Lockheed, he was with the MITRE Corporation, where he worked on the analysis and modeling of various digital radio communication systems. He then joined Lockheed Martin Global Telecommunications and worked on mobile satellite communication system analysis and design. From 1999-2012, he was with the School of Electrical Engineering and Computer Science, Ohio University, Athens, OH, and in 2012 he joined the Department of Electrical Engineering at the University of South Carolina. He has been a visiting professor at the National Institute of Standards and Technology, Boulder, CO, the University of Malaga, Spain, NASA Glenn Research Center, and the German Aerospace Center. He has done sponsored research for several national organizations, including DARPA/AFRL, NSF, NASA, NIST, and the FAA, and for L3 Communication Systems, and Texas Instruments. Dr. Matolak is a member of Eta Kappa Nu, Tau Beta Pi, Sigma Xi, the AAAS, the AIAA, the ASEE, and the URSI. He has served on dozens of IEEE Conference Technical Program committees, was also Chair of the Geo Mobile Radio Standards group in the Telecommunications Industries Association's (TIA's) Satellite Communications Division, and is a member of the ITU Radio Propagation Study Group 3. He is an editor for the IEEE Transactions on Vehicular Technology, and the IEEE Transactions on Wireless Communications. Research interests include radio channel modeling, modulation and detection, and mobile ad hoc networks. 


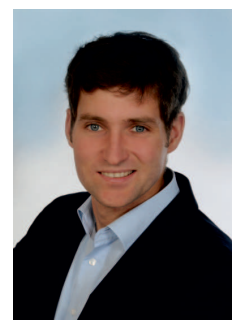

Nicolas Schneckenburger received his Dipl.-Ing. degree in electrical engineering from the University of Ulm in 2010 and his Dr.-Ing. from the Technical University of Ilmenau in 2017. Since 2010 he has been working as a research associate with the Institute of Communication and Navigation at DLR (German Aerospace Center). His main focus is on new communication systems in civil aviation and terrestrial navigation systems. In that context, he is concerned with modeling of the air to ground radio channel.

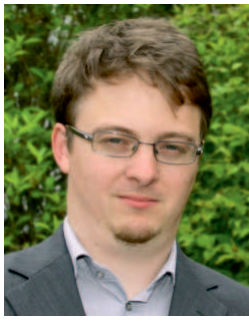

Thomas Wiedemann obtained a Bachelor and Master degree from the Faculty of Mechanical Engineering at the Technical University of Munich. $\mathrm{He}$ is a scientist at the Institute of Communications and Navigation, German Aerospace Center, where he is working in the Swarm Exploration Group since 2014. Further, he is enrolled as a PhD student at the AASS research center, Örebro University. His research interests include multi-robot systems, robotic exploration and Bayesian learning techniques.

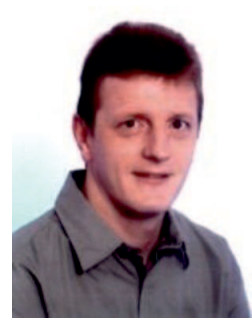

Armin Dammann received the Dipl.-Ing. (M.Sc.) and Dr.-Ing. (Ph.D.) degrees in Electrical Engineering from the University of Ulm, Germany, in 1997 and 2005 respectively. In 1997 he joined the Institute of Communications and Navigation of the German Aerospace Center (DLR). Since 2005 he is head of the Mobile Radio Transmission Research Group. His research interest and activities include signal design and signal processing for wireless communication and navigation systems. He has been active in several EU-projects, e.g., WINNER, WHERE and 